$\underline{\text { Preprint typeset in JHEP style - HYPER VERSION }}$

FTUAM 06/04

IFT-UAM/CSIC-06-19

KAIST-TH 06/07

\title{
Supersymmetry Breaking and Moduli Stabilization with Anomalous U(1) Gauge Symmetry
}

\author{
Kiwoon Choi ${ }^{a}$ and Kwang-Sik Jeong ${ }^{a, b}$ \\ ${ }^{a}$ Department of Physics, Korea Advanced Institute of Science and Technology \\ Daejeon 305-701, Korea \\ ${ }^{b}$ Departamento de Física Teórica $C$-XI and Instituto de Física Teórica $C-X V I$ \\ Universidad Autónoma de Madrid, Cantoblanco, E-28049 Madrid, Spain \\ E-mail: kchoi@hep.kaist.ac.kr, ksjeong@hep.kaist.ac.kr
}

\begin{abstract}
We examine the effects of anomalous $U(1)_{A}$ gauge symmetry on soft supersymmetry breaking terms while incorporating the stabilization of the modulus-axion multiplet responsible for the Green-Schwarz (GS) anomaly cancellation mechanism. In case of the KKLT stabilization of the GS modulus, soft terms are determined by the GS modulus mediation, the anomaly mediation and the $U(1)_{A}$ mediation which are generically comparable to each other, thereby yielding the mirage mediation pattern of superparticle masses at low energy scale. Independently of the mechanism of moduli stabilization and supersymmetry breaking, the $U(1)_{A} D$-term potential can not be an uplifting potential for de Sitter vacuum when the gravitino mass is smaller than the Planck scale by many orders of magnitude. We also discuss some features of the supersymmetry breaking by red-shifted anti-brane which is a key element of the KKLT moduli stabilization.
\end{abstract}

Keywords: Anomalous U(1) symmetry, Moduli Stabilization, Supersymmetry Breaking. 


\section{Contents}

1. Introduction 1

2. 4D effective action of KKLT compactification

3. Mass scales, $F$ and $D$ terms in 4D SUGRA with anomalous $U(1)$

4. A model for the KKLT stabilization of the GS modulus 19

5. Conclusion 26

\section{Introduction}

Anomalous $U(1)_{A}$ gauge symmetry appears often in compactified string theory. The 4dimensional (4D) spectrum of such compactification contains a modulus-axion (or dilatonaxion) superfield which transforms non-linearly under $U(1)_{A}$ to implement the GreenSchwarz (GS) anomaly cancellation mechanism [1]. In heterotic string theory, the dilaton plays the role of the GS modulus, however in other string theories, the GS modulus can be either a Kähler modulus of Calabi-Yau (CY) orientifold [2, 3] or a blowing-up modulus of orbifold singularity 唒. The non-linear $U(1)_{A}$ transformation of the GS modulus superfield leads to a field-dependent Fayet-Iliopoulos (FI) term [5] which might play an important role for supersymmetry (SUSY) breaking. Anomalous $U(1)_{A}$ might also correspond to a flavor symmetry which generates the hierarchical Yukawa couplings through the Froggatt-Nielsen mechanism [6, [].

The $U(1)_{A} D$-term can give a contribution to soft scalar masses as $\Delta m_{i}^{2}=-q_{i} g_{A}^{2} D_{A}$ where $q_{i}$ is the $U(1)_{A}$ charge of the corresponding sfermion [8]. Such $D$-term contribution has an important implication to the flavor problem in supersymmetric models. If $g_{A}^{2} D_{A}$ is significantly bigger than the gaugino mass-squares $M_{a}^{2}$ which are presumed to be of order $(1 \mathrm{TeV})^{2}$, e.g. $g_{A}^{2} D_{A} \sim(10 \mathrm{TeV})^{2}$, one can avoid the SUSY flavor problem by assuming that $q_{i}$ are non-vanishing only for the first and second generations of matter fields, which would make the first and second generations of squarks and sleptons heavy enough to avoid dangerous flavor-changing-neutral-current (FCNC) processes. Still one can arrange $q_{i}$ to be appropriately flavor-dependent [9] to generate the observed pattern of hierarchical Yukawa couplings via the Froggatt-Nielsen mechanism, e.g. $y_{i j} \sim \epsilon^{q_{i}+q_{j}}$ for $\epsilon \sim 0.2$. In other case that $g_{A}^{2} D_{A}$ is comparable to $M_{a}^{2}$, one needs $q_{i}$ to be flavor-universal to avoid dangerous FCNC processes, and then $U(1)_{A}$ can not be identified as a flavor symmetry for the Yukawa coupling hierarchy. Finally, if $g_{A}^{2} D_{A}$ is small enough, e.g. suppressed by a loop factor of order $10^{-2}$ compared to $M_{a}^{2}, q_{i}$ are again allowed to be flavor-dependent. It has been 
noticed that the relative importance of the $D$-term contribution to soft masses depends on how the GS modulus is stabilized [10]. In this respect, it is important to analyze the low energy consequences of anomalous $U(1)_{A}$ while incorporating the stabilization of the GS modulus explicitly [11, 12, 13].

In the previous studies of anomalous $U(1)_{A}$ in heterotic string compactification, two possible scenarios for the stabilization of the GS modulus (the heterotic string dilaton $S$ in this case) have been considered. One is to use the multiple gaugino condensations 14 which would stabilize $S$ at the weak coupling regime for which the leading order Kähler potential is a good approximation. In this race-track stabilization, one typically finds the auxiliary $F$ component $F^{S}=0$ and also $D_{A}=0$, although SUSY can be broken by the $F$-components of other moduli. The most serious difficulty of the race-track scenario is that in all known examples the vacuum energy density has a negative value of $\mathcal{O}\left(m_{3 / 2}^{2} M_{P l}^{2}\right)$ [15], where $M_{P l} \simeq 2.4 \times 10^{18} \mathrm{GeV}$ is the $4 \mathrm{D}$ reduced Planck mass and $m_{3 / 2}$ is the gravitino mass. Another possible scenario is that $S$ is stabilized by (presently not calculable) large quantum correction to the Kähler potential [16]. In this case, one can assume that the dilaton Kähler potential has a right form to stabilize $S$ at a phenomenologically viable de Sitter (dS) or Minkowski vacuum. The resulting $F^{S}$ and $D_{A}$ are non-vanishing in general, however the relative importance of $D_{A}$ compared to the other SUSY breaking auxiliary components depends sensitively on the incalculable large quantum corrections to the Kähler potential [10].

Recently a new way of stabilizing moduli at dS vacuum within a controllable approximation scheme has been proposed by Kachru-Kallosh-Linde-Trivedi (KKLT) in the context of Type IIB flux compactification [17. The main idea is to stabilize moduli (and also the dilaton) in the first step at a supersymmetric AdS vacuum for which the leading order Kähler potential is a good approximation, and then lift the vacuum to a dS state by adding anti-brane. For instance, in Type IIB compactification, one can first introduce a proper set of fluxes and gaugino condensations stabilizing all moduli at SUSY AdS vacuum. In the next step, anti-branes can be added to get the nearly vanishing cosmological constant under the RR charge cancellation condition. In the presence of fluxes, the compact internal space is generically warped [18] and anti-branes are stabilized at the maximally warped position [19]. Then as long as the number of anti-branes is small enough compared to the flux quanta, anti-branes cause neither a dangerous instability of the underlying compactification [19] nor a sizable shift of the moduli vacuum expectation values. In order to get the nearly vanishing cosmological constant, the anti-brane energy density should be adjusted to be close to $3 m_{3 / 2}^{2} M_{P l}^{2}$. This requires that the warp factor $e^{2 A}$ of the $4 \mathrm{D}$ metric on antibrane should be of $\mathcal{O}\left(m_{3 / 2} / M_{P l}\right)$. As it breaks explicitly the $N=1$ SUSY preserved by the background geometry and flux, one might expect that anti-brane will generate incalculable SUSY breaking terms in the low energy effective lagrangian. However as was noticed in 20] and will be discussed in more detail in this paper, the SUSY breaking soft terms in KKLT compactification can be computed within a reliable approximation scheme, which is essentially due to that anti-brane is red-shifted by a small warp factor $e^{2 A} \sim m_{3 / 2} / M_{P l}$.

In this paper, we wish to examine the implications of anomalous $U(1)_{A}$ for SUSY breaking while incorporating the stabilization of the GS modulus explicitly. Since one of 
our major concerns is the KKLT stabilization of the GS modulus, in section 2 we review the $4 \mathrm{D}$ effective action of KKLT compactification and discuss some features such as the $D$-type spurion dominance and the sequestering of the SUSY breaking by red-shifted anti-brane which is a key element of the KKLT compactification. In section 3, we discuss the mass scales, $F$ and $D$ terms in generic models of anomalous $U(1)_{A}$. In section 4 , we examine in detail a model for the KKLT stabilization of the GS modulus and the resulting pattern of soft terms. Section 5 is the conclusion.

The following is a brief summary of our results. The GS modulus-axion superfield $T$ transforms under $U(1)_{A}$ as

$$
T \rightarrow T-i \alpha(x) \frac{\delta_{G S}}{2}
$$

where $\alpha(x)$ is the $U(1)_{A}$ transformation function and $\delta_{G S}$ is a constant of $\mathcal{O}\left(1 / 8 \pi^{2}\right)$ when $T$ is normalized as $\partial_{T} f_{a}=\mathcal{O}(1)$ for the holomorphic gauge kinetic functions $f_{a}$. There are two mass scales that arise from the non-linear transformation of $T$ :

$$
\begin{gathered}
\xi_{F I}=\frac{\delta_{G S}}{2} \partial_{T} K_{0}, \\
M_{G S}^{2}=\frac{\delta_{G S}^{2}}{4} \partial_{T} \partial_{\bar{T}} K_{0},
\end{gathered}
$$

where $\xi_{F I}$ is the FI $D$-term and $M_{G S}^{2}$ corresponds to the GS axion contribution to the $U(1)_{A}$ gauge boson mass-square

$$
M_{A}^{2}=2 g_{A}^{2} M_{G S}^{2}+\mathcal{O}\left(\left|\xi_{F I}\right|\right)
$$

for the Kähler potential $K_{0}$ and the $U(1)_{A}$ gauge coupling $g_{A}$. (Unless specified, we will use the convention $M_{P l}=1$ throughout this paper.) Then the $U(1)_{A} D$-term is bounded as

$$
\left|D_{A}\right| \lesssim \mathcal{O}\left(m_{3 / 2}^{2} M_{P l}^{2} / M_{A}^{2}\right)
$$

for SUSY breaking scenarios with $m_{3 / 2} \ll M_{A}$.

It has been pointed out [2] that the $D$-term potential $V_{D}=\frac{1}{2} g_{A}^{2} D_{A}^{2}$ in models with anomalous $U(1)_{A}$ might play the role of an uplifting potential which compensates the negative vacuum energy density $-3 m_{3 / 2}^{2} M_{P l}^{2}$ in the supergravity potential. As the Kähler metric of $T$ typically has a vacuum expectation value of order unity, we have $M_{G S}^{2} \sim$ $M_{P l}^{2} /\left(8 \pi^{2}\right)^{2}$. Then, since the $U(1)_{A}$ gauge boson mass-square $M_{A}^{2} \gtrsim \mathcal{O}\left(M_{G S}^{2}\right)$, the above bound on $D_{A}$ implies that $V_{D}$ is too small to be an uplifting potential in SUSY breaking scenarios with $m_{3 / 2}<M_{P l} /\left(8 \pi^{2}\right)^{2}$. In other words, models of moduli stabilization in which $V_{D}$ plays the role of an uplifting potential for dS vacuum generically predict a rather large $m_{3 / 2} \gtrsim \mathcal{O}\left(M_{P l} /\left(8 \pi^{2}\right)^{2}\right)$ [13]. On the other hand, in view of that the gaugino masses receive the anomaly mediated contribution of $\mathcal{O}\left(m_{3 / 2} / 8 \pi^{2}\right)$, one needs $m_{3 / 2} \lesssim \mathcal{O}\left(8 \pi^{2}\right) \mathrm{TeV}$ in order to realize the supersymmetric extension of the standard model at the TeV scale. As a result, models with anomalous $U(1)_{A}$ still need an uplifting mechanism different from the 
$D$-term uplifting, e.g. the anti-brane uplifting of KKLT or a hidden matter superpotential suggested in [21], if $m_{3 / 2}$ is small enough to give the weak scale superparticle masses.

Still $D_{A}$ can give an important contribution to soft masses. As we will see, the relative importance of this $D$-term contribution depends on the size of the ratio

$$
R \equiv \xi_{F I} / M_{G S}^{2}
$$

If $\operatorname{Re}(T)$ is a string dilaton or a Kähler modulus which is stabilized at a vacuum expectation value of $\mathcal{O}(1)$ under the normalization $\partial_{T} f_{a}=\mathcal{O}(1)$, the resulting $|R|$ is of $\mathcal{O}\left(8 \pi^{2}\right)$. We then find the $D$-term contribution to soft masses is generically comparable to the GS modulus-mediated contribution. In this case of $|R| \gg 1$, the longitudinal component of the $U(1)_{A}$ gauge boson comes mostly from the phase of $U(1)_{A}$ charged field $X$ with a vacuum expectation value $\langle X\rangle \sim \sqrt{\xi_{F I}}$, rather than from the GS axion $\operatorname{Im}(T)$. Then $T$ is a flat-direction of the $U(1)_{A} D$-term potential, thus one needs a non-trivial $F$-term potential to stabilize $T$. An interesting possibility is the KKLT stabilization of $T$ involving a hidden gaugino condensation and also anti-brane for the uplifting mechanism. In such case, the soft terms are determined by three contributions mediated at the scales close to $M_{P l}$ : the GS modulus mediation [22], the anomaly mediation [23] and the $U(1)_{A}$ mediation [8]. Generically these three contributions are comparable to each other, yielding the mirage mediation pattern of superparticle masses at low energy scale [20, 24, 25, 26]. However if the Kähler potential of $X$ is related to the Kähler potential of $T$ in a specific manner, the $U(1)_{A}$ mediation is suppressed by a small factor of $\mathcal{O}\left(1 / 8 \pi^{2}\right)$ compared to the other two mediations. Since the anomaly mediation and the GS modulus mediation remain to be comparable to each other, the mirage mediation pattern is unaltered in this special case that the $U(1)_{A}$ mediation is relatively suppressed.

In fact, some models of anomalous $U(1)_{A}$ can yield $|R| \ll 1$. If $T$ corresponds to a blowing-up modulus of orbifold singularity stabilized at near the orbifold limit, one can have $\left|\xi_{F I}\right| \ll M_{G S}^{2}$ 四, 27], and thus $|R| \ll 1$. In this limit, soft terms mediated by the GS modulus at $M_{G S}$ are negligible compared to the soft terms mediated by a $U(1)_{A}$ charged

field $X$ at the lower scale $\langle X\rangle \sim \sqrt{\xi_{F I}}$. If $|R|$ is small enough, e.g. $|R| \lesssim 10^{-4}, U(1)_{A}$ $D$-term contribution is also smaller than the low scale mediation at $\sqrt{\xi_{F I}}$.

\section{2. $4 \mathrm{D}$ effective action of KKLT compactification}

In this section, we review the $4 \mathrm{D}$ effective action of KKLT compactification and the resulting soft SUSY breaking terms of visible fields. We also discuss some relevant features of the SUSY breaking by red-shifted anti-brane which is a key element of the KKLT compactification. KKLT compactification can be split into two parts. The first part contains the bulk of (approximate) CY space as well as the $D$ branes of visible matter and gauge fields which are assumed to be stabilized at a region where the warping is negligible. Note that the $4 \mathrm{D}$ cutoff scale of this part should be somewhat close to $M_{P l}$ in order to realize the $4 \mathrm{D}$ gauge coupling unification at $M_{G U T} \sim 2 \times 10^{16} \mathrm{GeV}$. The low energy dynamics of this part can be described by a $4 \mathrm{D}$ effective action which takes the form of conventional 
4D $N=1$ SUGRA:

$$
S_{\mathrm{N}=1}=\int d^{4} x d^{2} \Theta 2 \mathcal{E}\left[\frac{1}{8}\left(\overline{\mathcal{D}}^{2}-8 \mathcal{R}\right)\left(3 e^{-K / 3}\right)+\frac{1}{4} f_{a} W^{a \alpha} W_{\alpha}^{a}+W\right]+\text { h.c. },
$$

where $\Theta^{\alpha}$ is the Grassmann coordinate of the curved superspace, $\mathcal{E}$ is the chiral density, $\mathcal{R}$ is the chiral curvature superfield, and $K, f_{a}$ and $W$ denote the Kähler potential, gauge kinetic function and superpotential, respectively. In the following, we call this part the $N=1$ sector. The scalar potential of $S_{N=1}$ in the Einstein frame is given by

$$
V_{N=1}=e^{K}\left\{K^{I \bar{J}}\left(D_{I} W\right)\left(D_{J} W\right)^{*}-3|W|^{2}\right\}+\frac{1}{2 \operatorname{Re}\left(f_{a}\right)} D^{a} D^{a},
$$

where $D_{I} W=\partial_{I} W+\left(\partial_{I} K\right) W$ is the Kähler covariant derivative of the superpotential and $D^{a}=-\eta_{a}^{I} \partial_{I} K$ for the holomorphic Killing vector $\eta_{a}^{I}$ of the $a$-th gauge transformation of $\Phi^{I}$. In KKLT compactification, the $N=1$ sector is assumed to have a supersymmetric AdS vacuum ${ }^{1}$, i.e.

$$
\left\langle D_{I} W\right\rangle_{N=1}=0, \quad\left\langle V_{N=1}\right\rangle=-3 m_{3 / 2}^{2} M_{P l}^{2} .
$$

The remained part of KKLT compactification is anti-brane which is stabilized at the end of a warped throat. The SUSY preserved by anti-brane does not have any overlap with the $N=1$ SUSY preserved by the background geometry and flux. As a consequence, the field degrees of freedom on anti-brane do not have $N=1$ superpartner in general. For instance, the Goldstino fermion $\xi^{\alpha}$ of the broken $N=1$ SUSY which originates from anti-brane does not have bosonic $N=1$ superpartner. This means that the $N=1$ local SUSY is non-linearly realized on the world-volume of anti-brane. Still the anti-brane action can be written in a locally supersymmetric superspace form using the Goldstino superfield 28:

$$
\Lambda^{\alpha}=\xi^{\alpha}+\Theta^{\alpha}+\ldots
$$

where the ellipsis denotes the $\xi^{\alpha}$-dependent higher order terms. In the unitary gauge of $\xi^{\alpha}=0$, the anti-brane action appears to break the $N=1$ SUSY explicitly. Generic explicit SUSY breaking relevant for the soft terms of visible fields is described by three spurion operators: $D$-type spurion operator $\tilde{\mathcal{P}} \Theta^{2} \bar{\Theta}^{2}, F$-type non-chiral spurion operator $\tilde{\Gamma} \bar{\Theta}^{2}$, and $F$-type chiral spurion operator $\tilde{\mathcal{F}} \Theta^{2}$. Then the local lagrangian density on the world volume of anti-brane can be written as

$$
\begin{aligned}
\mathcal{L}_{\text {anti }}=\delta^{6}(y-\bar{y}) \int d^{2} \Theta 2 \mathcal{E}[ & \frac{1}{8}\left(\overline{\mathcal{D}}^{2}-8 \mathcal{R}\right)\left(e^{4 A} \tilde{\mathcal{P}} \Theta^{2} \bar{\Theta}^{2}+e^{3 A} \tilde{\Gamma} \bar{\Theta}^{2}\right) \\
& \left.-e^{4 A} \tilde{\mathcal{F}} \Theta^{2}+\ldots\right]+ \text { h.c. }
\end{aligned}
$$

where $\bar{y}$ is the coordinate of the anti-brane in six-dimensional internal space, $e^{2 A}$ is the warp factor on the anti-brane world volume:

$$
d s^{2}(\bar{y})=e^{2 A} g_{\mu \nu} d x^{\mu} d x^{\nu}
$$

\footnotetext{
${ }^{1}$ Note that $D^{a}=-\eta_{a}^{I} D_{I} W / W$, so $D_{I} W=0$ leads to $D^{a}=0$ for $W \neq 0$.
} 
and the ellipsis stands for the Goldstino-dependent terms which are not so relevant for us. Generically $\tilde{\mathcal{P}}, \tilde{\Gamma}$ and $\tilde{\mathcal{F}}$ have a value of order unity in the unit with $M_{P l}=1$ (or in the unit with the string scale $M_{\text {st }}=1$ ). The warp factor dependence of each spurion operator can be easily determined by noting that $\tilde{\mathcal{P}} \Theta^{2} \bar{\Theta}^{2}$ and $\tilde{\mathcal{F}} \Theta^{2}$ give rise to an anti-brane energy density which is red-shifted by $e^{4 A}$, while $\tilde{\Gamma} \bar{\Theta}^{2}$ gives rise to a gravitino mass on the antibrane world volume which is red-shifted by $e^{3 A}$. (See the discussion of Appendix A for this red-shift of gravitino mass.) Including the Goldstino fermion explicitly, the spurion operators in $\mathcal{L}_{\text {anti }}$ can be written in a locally supersymmetric form, e.g.

$$
\begin{aligned}
\tilde{\mathcal{P}} \Lambda^{2} \bar{\Lambda}^{2} & =\tilde{\mathcal{P}} \Theta^{2} \bar{\Theta}^{2}+\ldots, \\
\tilde{\Gamma} \bar{\Lambda}^{2} & =\tilde{\Gamma} \bar{\Theta}^{2}+\ldots, \\
\tilde{\mathcal{F}} \tilde{W}^{\alpha} \tilde{W}_{\alpha} & =\tilde{\mathcal{F}} \Theta^{2}+\ldots,
\end{aligned}
$$

where $\tilde{W}_{\alpha}=\frac{1}{8}\left(\overline{\mathcal{D}}^{2}-8 \mathcal{R}\right) \mathcal{D}_{\alpha}\left(\Lambda^{2} \bar{\Lambda}^{2}\right)$ and the ellipses denote the Goldstino-dependent terms.

The SUSY breaking spurions on the world volume of anti-brane can be transmitted to the visible $D$-branes by a bulk field propagating through the warped throat. The warp factor dependence of spurions allows us to estimate the size of SUSY breaking induced by each spurion without knowing the detailed mechanism of transmission. In addition to giving a vacuum energy density of $\mathcal{O}\left(e^{4 A} M_{P l}^{4}\right)$, the $D$-type spurion $\tilde{\mathcal{P}} \Theta^{2} \bar{\Theta}^{2}$ can generate SUSY breaking scalar mass-squares of $\mathcal{O}\left(e^{4 A} M_{P l}^{2}\right)$ through the effective operator $e^{4 A} \tilde{\mathcal{P}} \Theta^{2} \bar{\Theta}^{2} Q^{i *} Q^{i}$ which might be induced by the exchange of bulk fields, where $Q^{i}$ denote the visible matter superfields. The non-chiral $F$-type spurion $\tilde{\Gamma} \bar{\Theta}^{2}$ might generate trilinear scalar couplings of $\mathcal{O}\left(e^{3 A} M_{P l}\right)$ through the effective operator $e^{3 A} \tilde{\Gamma} \bar{\Theta}^{2} Q^{i *} Q^{i}$, while the chiral $F$-type spurion $\tilde{\mathcal{F}} \Theta^{2}$ might generate gaugino masses of $\mathcal{O}\left(e^{4 A} M_{P l}\right)$ through the effective chiral operator $e^{4 A} \tilde{\mathcal{F}} \Theta^{2} W^{a \alpha} W_{\alpha}^{a}$. When combined with its complex conjugate or with the $F$-component of $N=1$ sector moduli, $\tilde{\Gamma} \bar{\Theta}^{2}$ can generate a vacuum energy density of $\mathcal{O}\left(e^{6 A} M_{P l}^{4}\right)$ or $\mathcal{O}\left(e^{3 A} m_{3 / 2} M_{P l}^{3}\right)$, and scalar mass-squares of $\mathcal{O}\left(e^{6 A} M_{P l}^{2}\right)$ or $\mathcal{O}\left(e^{3 A} m_{3 / 2} M_{P l}\right)$. Similarly, the chiral $F$-type spurion $\tilde{\mathcal{F}} \Theta^{2}$ can generate a vacuum energy density and scalar masssquares, but they are suppressed by one more power of $e^{A}$ compared to the contribution from $\tilde{\Gamma} \bar{\Theta}^{2}$. In case with $e^{A} \sim 1$, all spurions give equally important contributions of the Planck scale size, leading to uncontrollable SUSY breaking. On the other hand, in case that $e^{A} \sim \sqrt{m_{3 / 2} / M_{P l}}$, which is in fact required in order for that the anti-brane energy density cancels the negative vacuum energy density (2.3) of the $N=1$ sector, SUSY breaking terms which originate from the $F$-type spurions are negligible compared to the terms which originate from the $D$-type spurion since they are suppressed by additional power of $e^{A} \sim \sqrt{m_{3 / 2} / M_{P l}}$. For instance, in the presence of the $D$-type spurion providing a vacuum energy density of $\mathcal{O}\left(m_{3 / 2}^{2} M_{P l}^{2}\right)$, there are always the anomaly-mediated soft masses of $\mathcal{O}\left(m_{3 / 2} / 8 \pi^{2}\right)$ which are much bigger than the soft masses induced by the $F$-type spurions when $e^{A} \ll 1 / 8 \pi^{2}$. Note that $e^{A} \sim \sqrt{m_{3 / 2} / M_{P l}} \lesssim 10^{-6}$ for $m_{3 / 2} \lesssim \mathcal{O}\left(8 \pi^{2}\right) \mathrm{TeV}$ which is necessary to get the weak scale SUSY. Obviously, this feature of $D$-type spurion dominance greatly simplifies the SUSY breaking by red-shifted anti-brane.

In addition to the Goldstino fermion, there can be other anti-brane fields, e.g. the anti- 
brane position moduli $\tilde{\phi}^{2}$ The anti-brane moduli also do not have $N=1$ superpartner, however one can construct the corresponding Goldstino-dependent superfields as

$$
\tilde{\Phi}=\tilde{\phi}+i\left(\Theta \sigma^{\mu} \bar{\xi}-\xi \sigma^{\mu} \bar{\Theta}\right) \partial_{\mu} \tilde{\phi}+\ldots
$$

The anti-brane lagrangian density including $\tilde{\Phi}$ and also the bulk moduli $\Phi$ which can have a local interaction on the world volume of anti-brane can be written as

$$
\mathcal{L}_{\text {anti }}=\delta^{6}(y-\bar{y}) \int d^{2} \Theta 2 e^{3 A} \mathcal{E}\left[\frac{1}{8} e^{-A}\left(\overline{\mathcal{D}}^{2}-8 \mathcal{R}\right) \Omega_{\text {anti }}\left(Z_{A}, Z_{A}^{*}\right)\right]+\text { h.c. },
$$

where $\Omega_{\text {anti }}$ is a function of $Z_{A}=\left\{e^{A / 2} \Lambda^{\alpha}, e^{-A / 2} \mathcal{D}_{\alpha}, e^{-A} \mathcal{R}, \tilde{\Phi}, \Phi\right\}$. Here the warp factor dependence of $\mathcal{L}_{\text {anti }}$ is determined by the Weyl weights of the involved superfields. Taking into account that the $F$-type spurions can be ignored in case of $e^{A} \sim \sqrt{m_{3 / 2} / M_{P l}}, \Omega_{\text {anti }}$ can be approximated as

$$
\Omega_{\mathrm{anti}} \simeq e^{2 A} \Lambda^{2} \bar{\Lambda}^{2}\left[\tilde{\mathcal{P}}\left(\Phi, \Phi^{*}\right)+\frac{1}{16} e^{-2 A} Z_{\tilde{\Phi}}\left(\Phi, \Phi^{*}\right) \tilde{\Phi}^{*} \overline{\mathcal{D}}^{2} \mathcal{D}^{2} \tilde{\Phi}+M_{\tilde{\Phi}}^{2}\left(\Phi, \Phi^{*}\right) \tilde{\Phi}^{*} \tilde{\Phi}\right]
$$

where $Z_{\tilde{\Phi}}=\mathcal{O}(1), M_{\tilde{\Phi}}=\mathcal{O}\left(M_{P l}\right)$, and $\langle\tilde{\Phi}\rangle$ is chosen to be zero. This shows that the antibrane moduli masses are generically of $\mathcal{O}\left(\sqrt{m_{3 / 2} M_{P l}}\right)$. Since it is confined on the world volume of anti-brane, $\tilde{\Phi}$ can not be a messenger of SUSY breaking, so can be integrated out without affecting the local SUSY breaking in the visible sector. Then, after integrating out the KK modes of bulk fields as well as the anti-brane moduli $\tilde{\Phi}$, the $4 \mathrm{D}$ effective action induced by $\Omega_{\text {anti }}$ takes the form:

$$
S_{\text {anti }}^{(4 D)}=\frac{1}{8} \int d^{4} x d^{2} \Theta 2 \mathcal{E}\left(\overline{\mathcal{D}}^{2}-8 \mathcal{R}\right)\left(\tilde{\mathcal{P}}\left(\Phi, \Phi^{*}\right)+\tilde{\mathcal{Y}}_{i}\left(\Phi, \Phi^{*}\right) Q^{i *} Q^{i}\right) e^{4 A} \Lambda^{2} \bar{\Lambda}^{2}+\text { h.c. }(2 .
$$

Note that the contact interaction between $e^{4 A} \Lambda^{2} \bar{\Lambda}^{2}$ and $Q^{i *} Q^{i}$ was not allowed in $\Omega_{\text {anti }}$ because $\Lambda^{\alpha}$ and the visible matter superfields $Q^{i}$ live on different branes which are geometrically separated from each other. Thus, if $\tilde{\mathcal{Y}}_{i} \neq 0$, it should be a consequence of the exchange of bulk fields which couple to both $e^{4 A} \Lambda^{2} \bar{\Lambda}^{2}$ (on anti-brane) and $Q^{i *} Q^{i}$ (on the $D$-branes of visible fields).

Possible phenomenological consequences of $S_{\text {anti }}^{(4 D)}$ are rather obvious. The Goldstino operator $e^{4 A} \tilde{\mathcal{P}} \Lambda^{2} \bar{\Lambda}^{2}$ gives rise to an uplifting potential of $\mathcal{O}\left(m_{3 / 2}^{2} M_{P l}^{2}\right)$ which would make the total vacuum energy density to be nearly vanishing. In the following, we will call this Goldstino operator the uplifting operator. ${ }^{3}$ The uplifting potential induces also a SUSY-breaking shift of the vacuum configuration (2.3), which would result in nonzero vacuum values of $F^{I}$ and $D^{a}$. The effective contact interaction between $Q^{i}$ and $\Lambda^{\alpha}$ gives soft SUSY-breaking sfermion mass-squares of $\mathcal{O}\left(\tilde{\mathcal{Y}}_{i} m_{3 / 2}^{2}\right)$. Note that the features of the $4 \mathrm{D}$ effective action of anti-brane which have been discussed so far rely only on that antibrane is red-shifted by the warp factor $e^{A} \sim \sqrt{m_{3 / 2} / M_{P l}}$, thus are valid for generic KKLT compactification.

\footnotetext{
${ }^{2}$ There can be also anti-brane gauge field $\tilde{A}_{\mu}$. However $\tilde{A}_{\mu}$ is not relevant for the transmission of SUSY breaking to the visible sector, thus will be ignored.

${ }^{3}$ In fact, this corresponds to the superspace expression of the Volkov-Akulov Goldstino lagrangian density.
} 
Since the scalar masses from the the effective contact term $e^{4 A} \Lambda^{2} \bar{\Lambda}^{2} Q^{i *} Q^{i}$ in $S_{\text {anti }}^{(4 D)}$ can be phenomenologically important, let us consider in what situation this contact interaction can be generated. The warped throat in KKLT compactification has approximately the geometry of $T_{5} \times \mathrm{AdS}_{5}$ where $T_{5}$ is a compact 5-manifold which is topologically $S^{2} \times S^{3}$. In the limit that the radius of $T_{5}$ is small compared to the length of the warped throat, the transmission of SUSY breaking through the throat can be described by a supersymmetric 5D Randall-Sundrum (RS) model [29] with visible $D$-branes at the UV fixed point $(y=0)$ and anti-brane at the IR fixed point $(y=\pi)$ [34. Let us thus examine the possible generation of the effective contact term within the framework of the supersymmetric 5D RS model.

It has been noticed that the 5D bulk SUGRA multiplet does not generate a contact interaction between UV superfield and IR superfield at tree level [30]. ${ }^{4}$ Loops of 5D SUGRA fields generate such contact interaction, however the resulting coefficient $\tilde{\mathcal{Y}}_{i}$ is suppressed by the warp factor $e^{2 A}$ [32], so is negligible. ${ }^{5}$ In fact, in order to generate the contact interaction $e^{4 A} \Lambda^{2} \bar{\Lambda}^{2} Q^{i *} Q^{i}$ in $4 \mathrm{D}$ effective action, one needs a bulk field $B$ other than the 5D SUGRA multiplet which has a non-derivative coupling in $N=1$ superspace to both $e^{4 A} \Lambda^{2} \bar{\Lambda}^{2}$ at the IR fixed point and $Q^{i *} Q^{i}$ at the UV fixed point. Since the SUGRA multiplet is not crucial for the following discussion, we will use the rigid $N=1$ superspace for simplicity, and then the required fixed point couplings of $B$ can be written as

$$
\int d^{2} \theta d^{2} \bar{\theta}\left[\delta(y) g_{B} B Q^{i *} Q^{i}+\delta(y-\pi) g_{B}^{\prime} e^{4 A} B \Lambda^{2} \bar{\Lambda}^{2}+\text { h.c. }\right],
$$

where $\theta^{\alpha}$ is the Grassmann coordinate of the rigid $N=1$ superspace. If $B$ is a chiral superfield in $N=1$ superspace, the effective contact interaction between $Q^{i *} Q^{i}$ and $e^{4 A} \Lambda^{2} \bar{\Lambda}^{2}$ induced by the exchange of $B$ is suppressed by the superspace derivative $\overline{\mathcal{D}}^{2}$. This can be easily noticed from the fact that the effective contact interaction arises from the part of the solution of $B$ which is proportional to the UV brane source $Q^{i *} Q^{i}$ or the IR brane source $e^{4 A} \Lambda^{2} \bar{\Lambda}^{2}$. Since the brane sources are non-chiral, this part of the solution should include the chiral projection operator $\overline{\mathcal{D}}^{2}$. As a result, the coefficient of the induced contact interaction is given by $\tilde{\mathcal{Y}}_{i} \sim g_{B} g_{B}^{\prime} \overline{\mathcal{D}}^{2} / k$ where $k$ is the AdS curvature which is essentially of $\mathcal{O}\left(M_{P l}\right)$. Since $\overline{\mathcal{D}}^{2} / k$ leads to an additional suppression by $m_{3 / 2} / M_{P l}$, the contact interaction induced by chiral bulk superfield gives at most a contribution of $\mathcal{O}\left(m_{3 / 2}^{3} / M_{P l}\right)$ to the soft scalar mass-squares of $Q^{i}$ when $e^{A} \sim \sqrt{m_{3 / 2} / M_{P l}}$, which is totally negligible.

On the other hand, if $B$ is a vector superfield in $N=1$ superspace, there is no such suppression by the chiral projection operator, so the resulting $\tilde{\mathcal{Y}}_{i}$ can be sizable in certain cases. To examine the contact term induced by a bulk vector superfield in more detail, one can consider the $5 \mathrm{D}$ lagrangian of $B$ which contains

$$
\int d^{2} \theta d^{2} \bar{\theta}\left[\frac{1}{8} B \mathcal{D}_{\alpha} \overline{\mathcal{D}}^{2} \mathcal{D}^{\alpha} B+M_{B}^{2} e^{-2 k L y} B^{2}\right.
$$

\footnotetext{
${ }^{4}$ In CFT interpretation, this might correspond to the conformal sequestering discussed in 31.

${ }^{5}$ Note the difference of the SUSY-breaking IR brane operator between our case and 32. In our case, the SUSY breaking IR brane operator is given by $e^{4 A} \Lambda^{2} \bar{\Lambda}^{2}$ for the Goldstino superfield $\Lambda^{\alpha}$ normalized as (2.4), while the SUSY breaking IR brane operator of [32] is $e^{2 A} Z^{*} Z$ for a $N=1$ chiral IR brane superfield $Z$ with nonzero $F^{Z}$.
} 


$$
\left.+\delta(y) g_{B} B Q^{i *} Q^{i}+\delta(y-\pi) g_{B}^{\prime} e^{-4 k L y} B \Lambda^{2} \bar{\Lambda}^{2}\right],
$$

where $e^{-k L y}$ is the position dependent warp factor in $\mathrm{AdS}_{5}, L$ is the orbifold length, and $M_{B}$ is the $5 \mathrm{D}$ mass of the vector superfield $B$. $\left(e^{-\pi k L}=e^{A}\right.$ in this convention.) The warp factor dependence of each term in the above $5 \mathrm{D}$ lagrangian can be determined by looking at the dependence on the background spacetime metric. Note that the UV brane coupling $g_{B}$ (the IR brane coupling $g_{B}^{\prime}$ ) corresponds to the gauge coupling between the $4 \mathrm{D}$ vector field component of $B$ and the $4 \mathrm{D}$ current component of the UV brane operator $Q^{i *} Q^{i}$ (the IR brane operator $\Lambda^{2} \bar{\Lambda}^{2}$ ). The $5 \mathrm{D}$ locality and dimensional analysis suggest that the contact term obtained by integrating out $B$ has a coefficient $\tilde{\mathcal{Y}}_{i} \propto e^{-\pi M_{B} L}$ in the limit $M_{B} \gg k$. Indeed, for $M_{B} \gtrsim k$, a more careful analysis [33] gives

$$
\tilde{\mathcal{Y}}_{i} \sim g_{B} g_{B}^{\prime} e^{-\pi\left(\sqrt{M_{B}^{2}+k^{2}}-k\right) L} / M_{B}^{2} .
$$

This result indicates that a sizable contact term can be induced if the model contains a vector superfield $B$ propagating through the warped throat with bulk mass $M_{B} \lesssim k$ and also sizable $g_{B}$ and $g_{B}^{\prime}$. In KKLT compactification of Type IIB string theory, one does not have such bulk vector superfield, thus it is expected that $\tilde{\mathcal{Y}}_{i}$ is negligibly small, i.e. anti-brane is sequestered well from the $D$-branes of visible fields. In fact, in KKLT compactification of Type IIB string theory, one finds that $\tilde{\mathcal{P}}$ is independent of the CY volume modulus [20], thus even the CY volume modulus is sequestered from anti-brane. This is not suprising in view of that the wavefunction of the volume modulus has a negligible value over the throat, thus the volume modulus can be identified as a UV brane field in the corresponding RS picture [34]. In the following, we will assume that $Q^{i}$ and $\Lambda^{\alpha}$ are sequestered from each other, thus

$$
\tilde{\mathcal{Y}}_{i}=0
$$

We stress that this sequestering assumption is relevant only for the soft scalar masses. The other SUSY breaking observables such as the gaugino masses and trilinear scalar couplings are not affected even when $\tilde{\mathcal{Y}}_{i}$ has a sizable value.

According to the above discussion, the $4 \mathrm{D}$ effective action of anti-brane is highly dominated by the uplifting operator:

$$
S_{\text {anti }}^{(4 D)} \simeq \frac{1}{8} \int d^{4} x d^{2} \Theta 2 \mathcal{E}\left(\overline{\mathcal{D}}^{2}-8 \mathcal{R}\right)\left(e^{4 A} \tilde{\mathcal{P}}\left(\Phi, \Phi^{*}\right) \Lambda^{2} \bar{\Lambda}^{2}\right)+\text { h.c. }
$$

and then the total 4D effective action of KKLT compactification is given by

$$
\begin{aligned}
S_{\mathrm{KKLT}}=S_{\mathrm{N}=1}+S_{\mathrm{anti}}^{(4 D)} & \\
=\int d^{4} x d^{2} \Theta 2 \mathcal{E}[ & \frac{1}{8}\left(\overline{\mathcal{D}}^{2}-8 \mathcal{R}\right)\left(3 e^{-K / 3}+\mathcal{P} \Lambda^{2} \bar{\Lambda}^{2}\right) \\
& \left.+\frac{1}{4} f_{a} W^{a \alpha} W_{\alpha}^{a}+W\right]+ \text { h.c. }
\end{aligned}
$$

where

$$
\mathcal{P}\left(\Phi, \Phi^{*}\right)=e^{4 A} \tilde{\mathcal{P}}\left(\Phi, \Phi^{*}\right)=\mathcal{O}\left(m_{3 / 2}^{2} M_{P l}^{2}\right)
$$


Since the vacuum expectation value of $\mathcal{P}$ can be fixed by the condition of vanishing cosmological constant, the above $4 \mathrm{D}$ effective action is almost equally predictive as the conventional $N=1$ SUGRA without the anti-brane term $\mathcal{P} \Lambda^{2} \bar{\Lambda}^{2}$. This nice feature of KKLT compactification is essentially due to that anti-brane is highly red-shifted.

In fact, for the discussion of moduli stabilization at a nearly flat $\mathrm{dS}$ vacuum and the subsequent SUSY breaking in the visible sector, the SUGRA multiplet can be simply replaced by their vacuum expectation values, e.g. $g_{\mu \nu}=\eta_{\mu \nu}$ and $\psi_{\mu}=0$, except for its scalar auxiliary component $\mathcal{M}$ whose vacuum expectation value should be determined by minimizing the scalar potential. The most convenient formulation for the SUSY breaking by $\mathcal{M}$ is to introduce the chiral compensator superfield $C$, then choose the superconformal gauge $\mathcal{M}=0$ to trade $\mathcal{M}$ for $F^{C}$, and finally replace the SUGRA multiplet by their vacuum values, while making the superconformal gauge choice in the rigid superspace:

$$
C=C_{0}+\theta^{2} F^{C} \text {. }
$$

In the unitary gauge, this procedure corresponds to the following replacements for the superspace action:

$$
\begin{aligned}
& \Lambda^{\alpha} \rightarrow \frac{C^{*}}{C^{1 / 2}} \theta^{\alpha}, \\
& W^{a \alpha} \rightarrow C^{-3 / 2} W^{a \alpha}, \\
& d^{2} \Theta 2 \mathcal{E} \rightarrow d^{2} \theta C^{3}, \\
& -\frac{1}{4} d^{2} \Theta 2 \mathcal{E}\left(\overline{\mathcal{D}}^{2}-8 \mathcal{R}\right) \rightarrow d^{2} \theta d^{2} \bar{\theta} C C^{*},
\end{aligned}
$$

under which the locally supersymmetric action (2.17) is changed to

$$
\begin{aligned}
S_{\mathrm{KKLT}}=\int d^{4} x & {\left[\int d^{2} \theta d^{2} \bar{\theta} C C^{*}\left(-3 e^{-K / 3}-C C^{*} \mathcal{P} \theta^{2} \bar{\theta}^{2}\right)\right.} \\
+ & \left.\left(\int d^{2} \theta\left(\frac{1}{4} f_{a} W^{a \alpha} W_{\alpha}^{a}+C^{3} W\right)+\text { h.c. }\right)\right] .
\end{aligned}
$$

Although written in the rigid superspace, the action (2.21) includes all SUGRA effects on SUSY breaking. Also as it has been derived from locally supersymmetric action without any inconsistent truncation, it provides a fully consistent low energy description of KKLT compactification which contains a red-shifted anti-brane. It is obvious that the uplifting anti-brane operator $\mathcal{P} \theta^{2} \bar{\theta}^{2}$ does not modify the solutions for the auxiliary components of $N=1$ superfields, thus

$$
\begin{aligned}
\frac{F^{C}}{C_{0}} & =\frac{1}{3} F^{I} \partial_{I} K+\frac{C_{0}^{* 2}}{C_{0}} e^{K / 3} W^{*}=\frac{1}{3} F^{I} \partial_{I} K+m_{3 / 2}^{*}, \\
F^{I} & =-\frac{C_{0}^{* 2}}{C_{0}} e^{K / 3} K^{I \bar{J}}\left(D_{J} W\right)^{*}=-e^{K / 2} K^{I \bar{J}}\left(D_{J} W\right)^{*}, \\
D^{a} & =-C_{0} C_{0}^{*} e^{-K / 3} \eta_{a}^{I} \partial_{I} K=-\eta_{a}^{I} \partial_{I} K,
\end{aligned}
$$

where $m_{3 / 2}=e^{K / 2} W$ and we have chosen the Einstein frame condition $C_{0}=e^{K / 6}$ for the last expressions. Here the index $I$ stands for generic chiral superfield $\Phi^{I}$, and $\eta_{a}^{I}$ is the 
holomorphic Killing vector for the infinitesimal gauge transformation:

$$
\delta_{a} \Phi^{I}=i \alpha_{a}(x) \eta_{a}^{I}
$$

Although it does not modify the on-shell expression of the auxiliary components of the $N=1$ superfields, the uplifting operator provides an additional scalar potential $V_{\text {lift }}$ which plays the role of an uplifting potential in KKLT compactification:

$$
V_{\mathrm{TOT}}=V_{F}+V_{D}+V_{\text {lift }},
$$

where

$$
\begin{aligned}
V_{F} & =\left(C_{0} C_{0}^{*}\right)^{2} e^{K / 3}\left\{K^{I \bar{J}}\left(D_{I} W\right)\left(D_{J} W\right)^{*}-3|W|^{2}\right\} \\
& =e^{K}\left\{K^{I \bar{J}}\left(D_{I} W\right)\left(D_{J} W\right)^{*}-3|W|^{2}\right\}, \\
V_{D} & =\frac{1}{2 \operatorname{Re}\left(f_{a}\right)} D^{a} D^{a}, \\
V_{\text {lift }} & =\left(C_{0} C_{0}^{*}\right)^{2} \mathcal{P}=e^{2 K / 3} \mathcal{P},
\end{aligned}
$$

where again the last expressions correspond to the results in the Einstein frame with $C_{0}=e^{K / 6}$.

Let us now consider the KKLT stabilization of CY moduli $\Phi$ and the resulting soft SUSY breaking terms of visible fields using the $4 \mathrm{D}$ effective action (2.17) or equivalently (2.21). In the first stage, $\Phi$ is stabilized at the SUSY AdS minimum $\Phi_{0}$ of $V_{N=1}=V_{F}+V_{D}$ for which

$$
D_{I} W\left(\Phi_{0}\right)=0, \quad W\left(\Phi_{0}\right) \neq 0 .
$$

The moduli masses at this SUSY AdS vacuum are dominated by the supersymmetric contribution which is presumed to be significantly larger than the gravitino mass:

$$
\left|m_{\Phi}\right| \gg\left|m_{3 / 2}\right|
$$

where

$$
m_{\Phi} \simeq-\left(\frac{e^{K / 2} \partial_{\Phi}^{2} W}{\partial_{\Phi} \partial_{\bar{\Phi}} K}\right)_{\Phi_{0}} .
$$

Adding the uplifting potential will shift the moduli vacuum values while making the total vacuum energy density to be nearly zero. Expanding the effective lagrangian of $\Phi$ around $\Phi_{0}$, one finds

$$
\begin{aligned}
\mathcal{L}_{\Phi}= & \partial_{\Phi} \partial_{\bar{\Phi}} K\left(\Phi_{0}\right)\left(\left|\partial_{\mu} \Delta \Phi\right|^{2}-\left|m_{\Phi}\right|^{2}|\Delta \Phi|^{2}\right)+3\left|m_{3 / 2}\right|^{2} M_{P l}^{2} \\
& -V_{\text {lift }}\left(\Phi_{0}\right)-\left(\Delta \Phi \partial_{\Phi} V_{\text {lift }}\left(\Phi_{0}\right)+\text { h.c }\right)+\ldots,
\end{aligned}
$$

where $\Delta \Phi=\Phi-\Phi_{0}$. Then the moduli vacuum shift is determined to be

$$
\frac{\Delta \Phi}{\Phi_{0}} \simeq-\frac{\Phi_{0}^{*} \partial_{\bar{\Phi}} V_{\mathrm{lift}}\left(\Phi_{0}\right)}{\left|\Phi_{0}\right|^{2} \partial_{\Phi} \partial_{\bar{\Phi}} K\left(\Phi_{0}\right)\left|m_{\Phi}\right|^{2}}=\mathcal{O}\left(\frac{m_{3 / 2}^{2}}{m_{\Phi}^{2}}\right)
$$


for $\left|\Phi_{0}\right|^{2} \partial_{\Phi} \partial_{\bar{\Phi}} K\left(\Phi_{0}\right)=\mathcal{O}(1)$ and $\Phi^{0} \partial_{\Phi} V_{\text {lift }}\left(\Phi_{0}\right)=\mathcal{O}\left(V_{\text {lift }}\left(\Phi_{0}\right)\right)=\mathcal{O}\left(m_{3 / 2}^{2} M_{P l}^{2}\right)$. This vacuum shift induces a nonzero $F^{\Phi}$ as

$$
\begin{aligned}
\frac{F^{\Phi}}{\Phi} & \simeq \frac{\Delta \Phi \partial_{\Phi} F^{\Phi}+\Delta \Phi^{*} \partial_{\bar{\Phi}} F^{\Phi}}{\Phi} \\
& \simeq-\frac{e^{K / 2}\left(\partial_{\Phi}^{2} W\right)^{*}}{\partial_{\Phi} \partial_{\bar{\Phi}} K} \frac{\Delta \Phi^{*}}{\Phi} \\
& \simeq-\left(\frac{3 \Phi^{*} \partial_{\Phi} \ln \left(V_{\text {lift }}\right)}{|\Phi|^{2} \partial_{\Phi} \partial_{\bar{\Phi}} K}\right) \frac{\left|m_{3 / 2}\right|^{2}}{m_{\Phi}}=\mathcal{O}\left(\frac{m_{3 / 2}^{2}}{m_{\Phi}}\right),
\end{aligned}
$$

where we have used $V_{\text {lift }}\left(\Phi_{0}\right) \simeq 3\left|m_{3 / 2}\right|^{2} M_{P l}^{2}$. The above result implies that heavy CY moduli with $m_{\Phi} \gg 8 \pi^{2} m_{3 / 2}$ are not relevant for the low energy SUSY breaking since the corresponding $F^{\Phi} / \Phi$ is negligible even compared to the anomaly mediated soft masses of $\mathcal{O}\left(m_{3 / 2} / 8 \pi^{2}\right)$.

In KKLT compactification, all complex structure moduli (and the Type IIB dilaton also) are assumed to get a heavy mass of $\mathcal{O}\left(M_{K K}^{3} / M_{\text {st }}^{2}\right)$ which is much heavier than $8 \pi^{2} m_{3 / 2}$ for $m_{3 / 2} \lesssim \mathcal{O}\left(8 \pi^{2}\right) \mathrm{TeV}$. (Here $M_{K K}$ and $M_{\text {st }}$ are the CY compactification scale and the string scale, respectively.) This means that complex structure moduli (and the Type IIB dilaton) are not relevant for the low energy soft terms, thus can be safely integrated out. On the other hand, the Kähler moduli masses from hidden gaugino condensations are given by $m_{\Phi} \sim m_{3 / 2} \ln \left(M_{P l}^{2} / m_{3 / 2}^{2}\right)$, and thus $F^{\Phi} / \Phi \sim m_{3 / 2} / 8 \pi^{2}$. As a result, the Kähler moduli can be an important messenger of SUSY breaking and generically their contributions to soft terms are comparable to the anomaly mediation [20].

The eqs. 2.30) and (2.31) show that one needs to know how the uplifting operator $\mathcal{P}$ depends on $\Phi$ in order to determine $F^{\Phi} / \Phi$. The above discussion implies also that only the dependence of $\mathcal{P}$ on the relatively light moduli with $m_{\Phi} \lesssim \mathcal{O}\left(8 \pi^{2} m_{3 / 2}\right)$ is relevant for the low energy SUSY breaking. In KKLT compactification of Type IIB string theory, anti-brane is stabilized at the end of a nearly collapsing 3-cycle. On the other hand, the messenger Kähler moduli correspond to the 4-cycle volumes, thus their wavefunctions have a negligible value at the end of the collapsing 3-cycle. This implies that Kähler moduli $\Phi$ are sequestered from anti-brane, i.e. $\partial_{\Phi} \ln \mathcal{P} \simeq 0$. Indeed, in this case, one finds $K=-3 \ln \left(T+T^{*}\right)$ and $V_{\text {lift }} \propto 1 /\left(T+T^{*}\right)^{2}$ for the $\mathrm{CY}$ volume modulus $T$, for which $\mathcal{P}=e^{-2 K / 3} V_{\text {lift }}$ is independent of $T$. On the other hand, in the absence of warped throat, one finds $V_{\text {lift }} \propto 1 /\left(T+T^{*}\right)^{3}$ and thus $\mathcal{P} \propto 1 /\left(T+T^{*}\right)$, showing that $T$ has a contact interaction with anti-brane. This indicates that the presence of warped throat is crucial for the sequestering as well as for the necessary red-shift of anti-brane. In this paper, although we are mainly interested in the sequestered anti-brane, we will leave it open possibility that $\mathcal{P}$ depends on some messenger moduli.

To derive the expression of the soft SUSY breaking terms of visible fields, let us expand $K$ and $W$ in powers of the visible chiral matter fields $Q^{i}$ :

$$
\begin{aligned}
K & =\mathcal{K}_{0}\left(\Phi^{x}, \Phi^{x *}, V\right)+Z_{i}\left(\Phi^{x}, \Phi^{x *}, V\right) Q^{i *} e^{2 q_{i} V} Q^{i}, \\
W & =W_{0}\left(\Phi^{x}\right)+\frac{1}{6} \tilde{\lambda}_{i j k}\left(\Phi^{x}\right) Q^{i} Q^{j} Q^{k},
\end{aligned}
$$


where $\Phi^{x}$ stand for generic messenger superfields of SUSY breaking, and $V$ is the vector superfield for gauge field. The soft SUSY breaking terms of canonically normalized visible fields can be written as

$$
\mathcal{L}_{\text {soft }}=-\frac{1}{2} M_{a} \lambda^{a} \lambda^{a}-\frac{1}{2} m_{i}^{2}\left|\tilde{Q}^{i}\right|^{2}-\frac{1}{6} A_{i j k} y_{i j k} \tilde{Q}^{i} \tilde{Q}^{j} \tilde{Q}^{k}+\text { h.c. },
$$

where $\lambda^{a}$ are gauginos, $\tilde{Q}^{i}$ is the scalar component of the superfield $Q^{i}$, and $y_{i j k}$ are the canonically normalized Yukawa couplings:

$$
y_{i j k}=\frac{\tilde{\lambda}_{i j k}}{\sqrt{e^{-\mathcal{K}_{0}} Z_{i} Z_{j} Z_{k}}} .
$$

Then from the superspace action (2.21), one finds that the soft masses renormalized at just below the GUT threshold scale $M_{G U T}$ are given by ${ }^{6}$

$$
\begin{aligned}
M_{a}= & F^{x} \partial_{x} \ln \left(\operatorname{Re}\left(f_{a}\right)\right)+\frac{b_{a} g_{a}^{2}}{8 \pi^{2}} \frac{F^{C}}{C_{0}}, \\
A_{i j k}= & -F^{x} \partial_{x} \ln \left(\frac{\tilde{\lambda}_{i j k}}{e^{-\mathcal{K}_{0}} Z_{i} Z_{j} Z_{k}}\right)-\frac{1}{16 \pi^{2}}\left(\gamma_{i}+\gamma_{j}+\gamma_{k}\right) \frac{F^{C}}{C_{0}}, \\
m_{i}^{2}= & \frac{2}{3}\left\langle V_{F}+V_{\text {lift }}\right\rangle-F^{x} F^{x *} \partial_{x} \partial_{\bar{x}} \ln \left(e^{-\mathcal{K}_{0} / 3} Z_{i}\right)-\left(q_{i}+\eta^{x} \partial_{x} \ln \left(Z_{i}\right)\right) g^{2}\langle D\rangle \\
& -\frac{1}{32 \pi^{2}} \frac{d \gamma_{i}}{d \ln \mu}\left|\frac{F^{C}}{C_{0}}\right|^{2}+\frac{1}{16 \pi^{2}}\left\{\left(\partial_{x} \gamma_{i}\right) F^{x}\left(\frac{F^{C}}{C_{0}}\right)^{*}+\text { h.c. }\right\} \\
= & \frac{2}{3}\left\langle V_{\text {lift }}\right\rangle+\left(\left\langle V_{F}\right\rangle+m_{3 / 2}^{2}-F^{x} F^{x *} \partial_{x} \partial_{\bar{x}} \ln \left(Z_{i}\right)\right)-\left(q_{i}+\eta^{x} \partial_{x} \ln \left(Z_{i}\right)\right) g^{2}\langle D\rangle \\
& -\frac{1}{32 \pi^{2}} \frac{d \gamma_{i}}{d \ln \mu}\left|\frac{F^{C}}{C_{0}}\right|^{2}+\frac{1}{16 \pi^{2}}\left\{\left(\partial_{x} \gamma_{i}\right) F^{x}\left(\frac{F^{C}}{C_{0}}\right)^{*}+\text { h.c. }\right\},
\end{aligned}
$$

where $\partial_{x}=\partial / \partial \Phi^{x}$ and $F^{x}$ is the $F$-component of $\Phi^{x}$ which can be determined by (2.30) and (2.31) in KKLT moduli stabilization scenario. Here we have included the anomaly mediated contributions, i.e. the parts involving $F^{C}$, and the $D$-term contribution (for $U(1)$ gauge group under which $\delta \Phi^{x}=i \alpha \eta^{x}$ ) as well as the contributions from $F^{x}$. As we will see in the next sections, all these three contributions can be comparable to each other in models with anomalous $U(1)_{A}$, thus should be kept altogether. Here $b_{a}$ and $\gamma_{i}$ are the one-loop beta function coefficients and the anomalous dimension of $Q^{i}$, respectively, defined by

$$
\frac{d g_{a}}{d \ln \mu}=\frac{b_{a}}{8 \pi^{2}} g_{a}^{3}, \quad \frac{d \ln Z_{i}}{d \ln \mu}=\frac{1}{8 \pi^{2}} \gamma_{i}
$$

More explicitly,

$$
b_{a}=-\frac{3}{2} \operatorname{tr}\left(T_{a}^{2}(\operatorname{Adj})\right)+\frac{1}{2} \sum_{i} \operatorname{tr}\left(T_{a}^{2}\left(Q^{i}\right)\right)
$$

\footnotetext{
${ }^{6}$ Note that these soft terms are the consequence of either a non-renormalizable interaction suppressed by $1 / M_{P l}$ or an exchange of messenger field with a mass close to $M_{P l}$. As a result, the messenger scale of these soft terms is close to $M_{P l}$ although the cutoff scale of the dynamical origin of SUSY breaking, i.e. the anti-brane, is $e^{A} M_{P l} \sim \sqrt{m_{3 / 2} M_{P l}}$ which is far below $M_{P l}$.
} 


$$
\begin{aligned}
\gamma_{i} & =2 C_{2}\left(Q^{i}\right)-\frac{1}{2} \sum_{j k}\left|y_{i j k}\right|^{2} \quad\left(\sum_{a} g_{a}^{2} T_{a}^{2}\left(Q^{i}\right) \equiv C_{2}\left(Q^{i}\right) \mathbf{1}\right), \\
\partial_{x} \gamma_{i} & =-\frac{1}{2} \sum_{j k}\left|y_{i j k}\right|^{2} \partial_{x} \ln \left(\frac{\tilde{\lambda}_{i j k}}{e^{-\mathcal{K}_{0}} Z_{i} Z_{j} Z_{k}}\right)-2 C_{2}\left(Q^{i}\right) \partial_{x} \ln \left(\operatorname{Re}\left(f_{a}\right)\right),
\end{aligned}
$$

where $\omega_{i j}=\sum_{k l} y_{i k l} y_{j k l}^{*}$ is assumed to be diagonal. Note that soft scalar masses depend on $\left\langle V_{\text {lift }}\right\rangle,\left\langle V_{F}\right\rangle$ and $\langle D\rangle$. Since any of $\left\langle V_{F}\right\rangle,\left\langle V_{\text {lift }}\right\rangle$ and $\langle D\rangle$ can give an important contribution to $m_{i}^{2}$ under the condition of vanishing cosmological constant:

$$
\left\langle V_{\text {TOT }}\right\rangle=\left\langle V_{F}\right\rangle+\left\langle V_{\text {lift }}\right\rangle+\left\langle V_{D}\right\rangle=0
$$

all of these contributions should be included with correct coefficients.

\section{Mass scales, $F$ and $D$ terms in 4D SUGRA with anomalous $U(1)$}

In this section, we discuss the mass scales and SUSY breaking $F$ and $D$ terms in $4 \mathrm{D}$ effective SUGRA which has an anomalous $U(1)_{A}$ gauge symmetry. To apply our results to the KKLT stabilization of the GS modulus, we will include the uplifting Goldstino superfield operator $\mathcal{P} \Lambda^{2} \bar{\Lambda}^{2}$ which was discussed in the previous section. The results for the conventional 4D SUGRA can be obtained by simply taking the limit $\mathcal{P}=0$.

In addition to the visible matter superfields $\left\{Q^{i}\right\}$, the model contains the MSSM singlet superfields $\left\{\Phi^{x}\right\}=\left\{T, X^{p}\right\}$ which can participate in SUSY breaking and/or $U(1)_{A}$ breaking, where $T$ is the GS modulus-axion superfield. These chiral superfields transform under $U(1)_{A}$ as

$$
U(1)_{A}: \quad \delta_{A} T=-i \alpha(x) \frac{\delta_{G S}}{2}, \quad \delta_{A} X^{p}=i \alpha(x) q_{p} X^{p}, \quad \delta_{A} Q^{i}=i \alpha(x) q_{i} Q^{i},
$$

where $\alpha(x)$ is the infinitesimal $U(1)_{A}$ transformation function, and $\delta_{G S}$ is a constant. We will choose the normalization of $T$ for which the holomorphic gauge kinetic functions are given by

$$
f_{a}=k_{a} T+T \text {-independent part, }
$$

where $k_{a}$ are real (quantized) constants of order unity. Under this normalization, we need $|\langle T\rangle| \lesssim \mathcal{O}(1)$ to get the gauge coupling constants of order unity, and also the cancellation of anomalies by the $U(1)_{A}$ variation of $k_{a} \operatorname{Im}(T) F^{a \mu \nu} \tilde{F}_{\mu \nu}^{a}$ requires

$$
\delta_{G S}=\mathcal{O}\left(\frac{1}{8 \pi^{2}}\right)
$$

Models with anomalous $U(1)_{A}$ gauge symmetry contain also an approximate global $U(1)$ symmetry:

$$
U(1)_{T}: \quad \delta_{T} T=i \beta, \quad \delta_{T} X^{p}=\delta_{T} Q^{i}=0,
$$


where $\beta$ is an infinitesimal constant. Obviously $U(1)_{T}$ is explicitly broken by $\delta_{T} f_{a}=i k_{a} \beta$ as well as by non-perturbative effects depending on $e^{-c T}$ with an appropriate constant $c$. In some cases, it is more convenient to consider the following approximate global symmetry

$$
U(1)_{X}: \quad \delta_{X} T=0, \quad \delta_{X} X^{p}=i \beta q_{p} X^{p}, \quad \delta_{X} Q^{i}=i \beta q_{i} Q^{i}
$$

which is a combination of $U(1)_{A}$ and $U(1)_{T}$. The fact that quantum amplitudes are free from $U(1)_{A}$ anomaly requires

$$
\left(\delta_{X} f_{a}\right)_{1-\mathrm{loop}}=i \beta k_{a} \frac{\delta_{G S}}{2},
$$

where $\delta_{X} f_{a}$ represent the $U(1)_{X}$ anomalies due to the fermion loops.

For generic 4D SUGRA action (2.17) including the Goldstino superfield operator $\mathcal{P} \Lambda^{2} \bar{\Lambda}^{2}$, one can find the following relation between the vacuum expectation values of SUSY breaking quantities:

$$
\begin{aligned}
& \left(V_{F}+\frac{2}{3} V_{\text {lift }}+2\left|m_{3 / 2}\right|^{2}+\frac{1}{2} M_{A}^{2}\right) D_{A} \\
& =-F^{I} F^{J *} \partial_{I}\left(\eta^{L} \partial_{L} \partial_{\bar{J}} K\right)+V_{D} \eta^{I} \partial_{I} \ln g_{A}^{2}+V_{\text {lift }} \eta^{I} \partial_{I} \ln \mathcal{P},
\end{aligned}
$$

where $g_{A}, D_{A}, M_{A}$ and $\eta^{I}$ denote the gauge coupling, $D$-term, gauge boson mass, and holomorphic Killing vector of $U(1)_{A}$, respectively:

$$
D_{A}=-\eta^{I} \partial_{I} K, \quad M_{A}^{2}=2 g_{A}^{2} \eta^{I} \eta^{J *} \partial_{I} \partial_{\bar{J}} K
$$

for the $U(1)_{A}$ transformation $\delta_{A} \Phi^{I}=i \alpha(x) \eta^{I}$. Here $V_{F}, V_{D}$ and $V_{\text {lift }}$ are the $F$-term potential, the $D$-term potential and the uplifting potential, respectively:

$$
\begin{aligned}
& V_{F}=K_{I \bar{J}} F^{I} F^{J *}-3\left|m_{3 / 2}\right|^{2}, \\
& V_{D}=\frac{1}{2} g_{A}^{2} D_{A}^{2}, \quad V_{\text {lift }}=e^{2 K / 3} \mathcal{P},
\end{aligned}
$$

and all quantities are evaluated for the vacuum configuration satisfying

$$
\partial_{I} V_{\mathrm{TOT}}=\partial_{I}\left(V_{F}+V_{D}+V_{\text {lift }}\right)=0 .
$$

The relation (3.7) has been derived before [35] for the conventional 4D SUGRA without $V_{\text {lift }}$. Since it plays an important role for our subsequent discussion, let us briefly sketch the derivation of (3.7) for SUGRA including the uplifting operator $\mathcal{P} \Lambda^{2} \bar{\Lambda}^{2}$. From the $U(1)_{A}$ invariances of $K$ and $W$, one easily finds

$$
\eta^{I} \partial_{I} K=\eta^{I *} \partial_{\bar{I}} K, \quad \eta^{I} D_{I} W=-W D_{A}
$$

which lead to

$$
\begin{aligned}
& \eta^{I} \partial_{I} D_{A}=-\eta^{I} \eta^{J *} \partial_{I} \partial_{\bar{J}} K=-\frac{M_{A}^{2}}{2 g_{A}^{2}}, \\
& \left(\partial_{L} \eta^{I}\right) D_{I} W+\eta^{I} \partial_{I}\left(D_{L} W\right)=W \eta^{\bar{I}} \partial_{\bar{I}} \partial_{L} K .
\end{aligned}
$$


Using these relations, one can find

$$
\begin{aligned}
\eta^{I} \partial_{I} V_{D} & =V_{D} \eta^{I} \partial_{I} \ln \left(g_{A}^{2}\right)-\frac{1}{2} M_{A}^{2} D_{A}, \\
\eta^{I} \partial_{I} V_{F} & =-\left(V_{F}+2\left|m_{3 / 2}\right|^{2}\right) D_{A}-F^{I} F^{J *} \partial_{I}\left(\eta^{L} \partial_{L} \partial_{\bar{J}} K\right), \\
\eta^{I} \partial_{I} V_{\text {lift }} & =\left(-\frac{2}{3} D_{A}+\eta^{I} \partial_{I} \ln (\mathcal{P})\right) V_{\text {lift. }} .
\end{aligned}
$$

Applying the stationary condition (3.10) to (3.13), one finally obtains the relation (3.7).

For the analysis of SUSY and $U(1)_{A}$ breaking, we can simply set $Q^{i}=0$. Also for simplicity, we assume that all moduli other than $T$ can be integrated out without affecting the SUSY and $U(1)_{A}$ breaking. Then $X^{p}$ correspond to the $U(1)_{A}$ charged but MSSM singlet chiral superfields with vacuum expectation values which are small enough to allow the expansion in powers of $X^{p} / M_{P l}$, but still large enough to play an important role in SUSY and/or $U(1)_{A}$ breaking. To be concrete, we will use the Kähler potential which takes the form:

$$
\begin{aligned}
K & =\mathcal{K}_{0}\left(\Phi^{x}, \Phi^{x *}, V_{A}\right)+Z_{i}\left(t_{V}\right) Q^{i *} e^{2 q_{i} V_{A}} Q^{i} \\
& =K_{0}\left(t_{V}\right)+Z_{p}\left(t_{V}\right) X^{p *} e^{2 q_{p} V_{A}} X^{p}+Z_{i}\left(t_{V}\right) Q^{i *} e^{2 q_{i} V_{A}} Q^{i}
\end{aligned}
$$

where $t_{V}=T+T^{*}-\delta_{G S} V_{A}$ for the $U(1)_{A}$ vector superfield $V_{A}$, however our results will be valid for more general $K$ including the terms higher order in $X^{p} / M_{P l}$. For the above Kähler potential, the $U(1)_{A} D$-term and gauge boson mass-square are given by

$$
\begin{aligned}
D_{A} & =\xi_{F I}-q_{p} \tilde{Z}_{p}\left|X^{p}\right|^{2}, \\
\frac{M_{A}^{2}}{2 g_{A}^{2}} & =M_{G S}^{2}+\left(q_{p}^{2} \tilde{Z}_{p}-\frac{\delta_{G S}}{2} q_{p} \partial_{T} \tilde{Z}_{p}\right)\left|X^{p}\right|^{2},
\end{aligned}
$$

where $\xi_{F I}$ and $M_{G S}^{2}$ are the FI $D$-term and the GS axion contribution to $M_{A}^{2}$, respectively:

$$
\begin{gathered}
\xi_{F I}=\frac{\delta_{G S}}{2} \partial_{T} K_{0}, \\
M_{G S}^{2}=\frac{\delta_{G S}^{2}}{4} \partial_{T} \partial_{\bar{T}} K_{0},
\end{gathered}
$$

and

$$
q_{p} \tilde{Z}_{p}=q_{p} Z_{p}-\frac{\delta_{G S}}{2} \partial_{T} Z_{p}
$$

If $|\langle T\rangle| \lesssim \mathcal{O}(1)$ as required for the gauge coupling constants to be of order unity, the Kähler metric of $T$ is generically of order unity, and then

$$
M_{G S} \sim \delta_{G S} M_{P l} \sim \frac{M_{P l}}{8 \pi^{2}}
$$

On the other hand, the size of $\xi_{F I}$ depends on the more detailed property of $T$. If $\operatorname{Re}(T)$ is a dilaton or a Kähler modulus stabilized at $\langle\operatorname{Re}(T)\rangle=\mathcal{O}(1)$, we have $\left|\xi_{G S}\right| \simeq$ $M_{G S}^{2}\left(T+T^{*}\right) /\left|\delta_{G S}\right| \sim 8 \pi^{2} M_{G S}^{2}$. In another case that $T$ is a blowing-up modulus of orbifold singularity stabilized at near the orbifold limit, the resulting $\left|\xi_{F I}\right| \ll M_{G S}^{2}$. 
In view of that the gaugino masses receive the anomaly mediated contribution of $\mathcal{O}\left(m_{3 / 2} / 8 \pi^{2}\right)$, one needs $m_{3 / 2}$ hierarchically lower than $M_{P l}$, e.g. $m_{3 / 2} \lesssim \mathcal{O}\left(8 \pi^{2}\right) \mathrm{TeV}$, in order to realize the supersymmetric extension of the standard model at the TeV scale. Since the $U(1)_{A}$ gauge boson mass is always rather close to $M_{P l}$ :

$$
M_{A} \gtrsim \sqrt{2} g_{A} M_{G S} \sim \frac{M_{P l}}{8 \pi^{2}},
$$

let us focus on models with

$$
m_{3 / 2} \ll M_{A}, \quad\left\langle V_{\mathrm{TOT}}\right\rangle \simeq 0
$$

and examine the mass scales in such models. The condition of nearly vanishing cosmological constant requires that

$$
K_{I \bar{J}} F^{I} F^{J *} \lesssim \mathcal{O}\left(m_{3 / 2}^{2} M_{P l}^{2}\right), \quad V_{\text {lift }} \lesssim \mathcal{O}\left(m_{3 / 2}^{2} M_{P l}^{2}\right),
$$

and then the relation (3.7) implies

$$
\left|D_{A}\right| \lesssim \mathcal{O}\left(\frac{m_{3 / 2}^{2} M_{P l}^{2}}{M_{A}^{2}}\right) \lesssim \mathcal{O}\left(\left(8 \pi^{2}\right)^{2} m_{3 / 2}^{2}\right) .
$$

It has been pointed out that one might not need to introduce anti-brane to obtain a dS vacuum if the $D$-term potential $V_{D}=\frac{1}{2} g_{A}^{2} D_{A}^{2}$ can compensate the negative vacuum energy density $-3 m_{3 / 2}^{2} M_{P l}^{2}$ in $V_{F}$ [2]. The second relation of (3.11) indicates that $F^{I} \neq 0$ is required for $D_{A} \neq 0$, thus the $D$-term uplifting scenario can not be realized for the supersymmetric AdS solution of $V_{F}$. However for a SUSY-breaking solution with $F^{I} \neq 0$, $V_{D}$ might play the role of an uplifting potential making $\left\langle V_{F}+V_{D}\right\rangle \geq 0$. The above bound on $D_{A}$ imposes a severe limitation on such possibility as it implies that $V_{D}$ can not be an uplifting potential in SUSY breaking scenarios with $m_{3 / 2} \ll M_{A}^{2} / M_{P l}$. In other words, SUSY breaking models in which $V_{D}$ plays the role of an uplifting potential generically predict a rather large $m_{3 / 2} \gtrsim \mathcal{O}\left(M_{A}^{2} / M_{P l}\right) \gtrsim \mathcal{O}\left(M_{P l} /\left(8 \pi^{2}\right)^{2}\right)$. For instance, the model of 13] in which $V_{D}$ indeed compensates $-3 m_{3 / 2}^{2} M_{P l}^{2}$ in $V_{F}$ gives $M_{A}=\mathcal{O}\left(M_{P l} / \sqrt{8 \pi^{2}}\right)$ and $m_{3 / 2}=\mathcal{O}\left(M_{P l} / 8 \pi^{2}\right)$.

Let us now examine more detailed relations between the $F$ and $D$ terms for the Kähler potential (3.14). In case that $\langle\operatorname{Re}(T)\rangle=\mathcal{O}(1)$, the FI $D$-term is rather close to $M_{P l}^{2}$ : $\xi_{F I}=\mathcal{O}\left(M_{P l}^{2} / 8 \pi^{2}\right)$. Such a large value of $\xi_{F I}$ in $D_{A}$ should be cancelled by $q_{p} \tilde{Z}_{p}\left|X^{p}\right|^{2}$ in order to give $D_{A}$ satisfying the bound (3.22), thus

$$
\xi_{F I} \simeq q_{p} \tilde{Z}_{p}\left|X^{p}\right|^{2}
$$

In some case, for instance the case that the GS modulus is a blowing-up mode of orbifold singularity, $\xi_{F I}$ can have a vacuum value smaller than $M_{P l}^{2}$ by many orders of magnitude. However the existence of the anomalous (approximate) global symmetry $U(1)_{X}$ implies that some $X^{p}$ should get a large vacuum value $\left|X^{p}\right|^{2} \gg\left|D_{A}\right|$ to break $U(1)_{X}$ at a sufficiently high energy scale. This means that $\left|\xi_{F I}\right| \gg\left|D_{A}\right|$ and the relation (3.23) remains to be 
valid even in case that $\left|\xi_{F I}\right|$ is smaller than $M_{P l}^{2}$ by many orders of magnitude. Then using $\eta^{I} D_{I} W=-W D_{A}$, we find

$$
\begin{aligned}
F^{T} & =\frac{q_{p} \tilde{Z}_{p}\left|X^{p}\right|^{2}}{\delta_{G S} \partial_{T} \partial_{\bar{T}} K_{0} / 2-q_{r} \partial_{T} \tilde{Z}_{r}\left|X^{r}\right|^{2}}\left(\frac{F^{p}}{X^{p}}\right)+\mathcal{O}\left(\frac{8 \pi^{2} m_{3 / 2} D_{A}}{M_{P l}}\right) \\
& =\frac{\mathcal{O}\left(\delta_{G S} \xi_{F I}\right)}{M_{G S}^{2}+\mathcal{O}\left(\delta_{G S} \xi_{F I}\right)} \frac{F^{p}}{X^{p}},
\end{aligned}
$$

where we have used (3.23) for the last expression. Applying this relation to (3.7), we also find

$$
\begin{aligned}
g_{A}^{2} D_{A}= & -\frac{q_{p} \tilde{Z}_{p} \delta_{p \bar{q}}+q_{p} q_{q} X^{p *} X^{q} \partial_{T}\left[\tilde{Z}_{p} \tilde{Z}_{q} /\left(\delta_{G S} \partial_{T} \partial_{\bar{T}} K_{0} / 2-q_{r} \partial_{T} \tilde{Z}_{r}\left|X^{r}\right|^{2}\right)\right]}{\delta_{G S}^{2} \partial_{T} \partial_{\bar{T}} K_{0} / 4+\left(q_{r}^{2} \tilde{Z}_{r}-q_{r} \delta_{G S} \partial_{T} \tilde{Z}_{r} / 2\right)\left|X^{r}\right|^{2}} F^{p} F^{q *} \\
& +\frac{V_{\text {lift }} \eta^{I} \partial_{I} \ln \mathcal{P}}{\delta_{G S}^{2} \partial_{T} \partial_{\bar{T}} K_{0} / 4+\left(q_{r}^{2} \tilde{Z}_{r}-q_{r} \delta_{G S} \partial_{T} \tilde{Z}_{r} / 2\right)\left|X^{r}\right|^{2}} \\
= & \frac{\mathcal{O}\left(\xi_{F I}\right)}{M_{G S}^{2}+\mathcal{O}\left(\xi_{F I}\right)}\left|\frac{F^{p}}{X^{p}}\right|^{2}+\frac{V_{\text {lift }}}{M_{G S}^{2}+\mathcal{O}\left(\xi_{F I}\right)} \eta^{I} \partial_{I} \ln \mathcal{P} .
\end{aligned}
$$

Note that the piece proportional to $V_{\text {lift }}$ vanishes if the Goldstino superfield on anti-brane is sequestered from the $U(1)_{A}$ charged fields, i.e. $\eta^{I} \partial_{I} \mathcal{P}=0$, which is a rather plausible possibility in view of our discussion in section 2 .

The relations (3.24) and (3.25) show that the relative importance of the GS modulus mediation and the $U(1)_{A} D$-term mediation is determined essentially by the ratio

$$
R \equiv \frac{\xi_{F I}}{M_{G S}^{2}}=\frac{2 \partial_{T} K_{0}}{\delta_{G S} \partial_{T} \partial_{\bar{T}} K_{0}}
$$

If $T$ is a string dilaton or a Kähler modulus normalized as $\partial_{T} f_{a}=\mathcal{O}(1)$, its Kähler potential is given by

$$
K_{0}=-n_{0} \ln \left(T+T^{*}\right)+\mathcal{O}\left(1 / 8 \pi^{2}\left(T+T^{*}\right)\right) .
$$

As long as $\operatorname{Re}(T)$ is stabilized at a value of $\mathcal{O}(1)$, the higher order string loop or $\alpha^{\prime}$ corrections to $K_{0}$ can be safely ignored, yielding $|R|=\mathcal{O}\left(8 \pi^{2}\right)$. In such case, (3.24) and (3.25) imply that generically

$$
\left|D_{A}\right| \sim\left|F^{T}\right|^{2} \sim\left|\frac{F^{p}}{X^{p}}\right|^{2} .
$$

Note that in the limit $|R| \gg 1$, the $U(1)_{A}$ gauge boson mass-square is dominated by the contribution from $\left\langle X^{p}\right\rangle \sim \sqrt{\left|\xi_{F I}\right|}$. In this case, the longitudinal component of the massive $U(1)_{A}$ gauge boson comes mostly from the phase degrees of $X^{p}$, while the GS modulus $T$ is approximately a flat direction of the $U(1)_{A} D$-term potential. An interesting possibility is then to stabilize $T$ by non-perturbative superpotential at a SUSY AdS vacuum with $\operatorname{Re}(T)=\mathcal{O}(1)$, and then lift this AdS vacuum to dS state by adding a red-shifted antibrane as in the KKLT moduli stabilization scenario. In the next section, we will discuss such KKLT stabilization of the GS modulus in more detail together with the resulting pattern of soft SUSY breaking terms. 
Another possibility is that $\operatorname{Re}(T)$ is a blowing-up modulus of orbifold singularity, thus $\xi_{F I}=\delta_{G S} \partial_{T} K_{0}=0$ in the orbifold limit. Choosing $\operatorname{Re}(T)=0$ in the orbifold limit, $K_{0}$ can be expanded as

$$
K_{0} \approx \frac{1}{2} a_{0}\left(T+T^{*}\right)^{2}+\mathcal{O}\left(\left(T+T^{*}\right)^{3}\right)
$$

for a constant $a_{0}$. If $\operatorname{Re}(T)$ is stabilized at near the orbifold limit for which $\left|\xi_{F I}\right| \ll M_{G S}^{2}$, the resulting $|R| \ll 1$. In this limit, if the uplifting anti-brane is sequestered from the $U(1)_{A}$ charged fields, i.e. $\eta^{I} \partial_{I} \mathcal{P}=0$, eqs. (3.24) and (3.25) lead to

$$
F^{T} \sim \delta_{G S} R \frac{F^{p}}{X^{p}}, \quad D_{A} \sim R\left|\frac{F^{p}}{X^{p}}\right|^{2},
$$

where $F^{p} / X^{p}$ represents the SUSY breaking mediated at the scale around $\left\langle X^{p}\right\rangle \sim \sqrt{\left|\xi_{F I}\right|} \ll$ $M_{G S}$. The anomaly condition (3.6) for the $U(1)_{X}$ symmetry (3.5) implies that the gauge kinetic functions receive a loop correction $\Delta f_{a} \sim \frac{1}{8 \pi^{2}} \ln X^{p}$ at the scale $\left\langle X^{p}\right\rangle$ where $U(1)_{X}$ is spontaneously broken. For instance, there might be a coupling $X^{p} Q_{1} Q_{2}$ in the superpotential generating $\Delta f_{a}$ through the loop of $Q_{1}+Q_{2}$ which are charged under the standard model gauge group. ${ }^{7}$ This results in the gaugino masses

$$
M_{a}=\mathcal{O}\left(\frac{1}{8 \pi^{2}} \frac{F^{p}}{X^{p}}\right)
$$

mediated at the scale $\left\langle X^{p}\right\rangle$. Obviously $F^{T}$ is smaller than this $M_{a}$ in the limit $|R| \ll 1$. If $\xi_{F I}$ is smaller than $M_{P l}^{2}$ by many orders of magnitude, e.g. $|R| \lesssim 10^{-4},\left|D_{A}\right|$ also is smaller than $\left|M_{a}\right|^{2}$ mediated at $\left\langle X^{p}\right\rangle$. Then the soft terms are dominated by the contributions mediated at the low messenger scale around $\left\langle X^{p}\right\rangle \sim \sqrt{\left|\xi_{F I}\right|}$. Those soft terms with low messenger scale depend on more detailed property of the model, which is beyond the scope of this paper.

\section{A model for the KKLT stabilization of the GS modulus}

In this section, we discuss a model for the KKLT stabilization of the GS modulus $T$ in detail. In this model, $T$ is stabilized at a value of $\mathcal{O}(1)$, yielding $\xi_{F I} \sim \delta_{G S} M_{P l}^{2}$. For simplicity, we introduce a single $U(1)_{A}$ charged MSSM singlet $X$ whose vacuum value cancels $\xi_{F I}$ in $D_{A}$. In addition to $X$ and the visible matter superfields $Q^{i}$, one needs also a hidden $S U\left(N_{c}\right)$ Yang-Mills sector with $S U\left(N_{c}\right)$ charged matter fields $Q_{H}+Q_{H}^{c}$ in order to produce non-perturbative superpotential stabilizing $T$. The gauge kinetic functions of the model are given by

$$
f_{a}=k T+\Delta f, \quad f_{H}=k_{H} T+\Delta f_{H},
$$

where $f_{a}(a=3,2,1)$ and $f_{H}$ are the gauge kinetic functions of the $S U(3)_{c} \times S U(2)_{W} \times$ $U(1)_{Y}$ and the hidden $S U\left(N_{c}\right)$ gauge group, respectively, and $k$ and $k_{H}$ are real constants of $\mathcal{O}(1)$. Generically $\Delta f$ and $\Delta f_{H}$ can depend on other moduli of the model. Here we

\footnotetext{
${ }^{7}$ This corresponds to the gauge mediation at the messenger scale $\left\langle X^{p}\right\rangle$.
} 
assume that those other moduli are fixed by fluxes with a large mass $\gg 8 \pi^{2} m_{3 / 2}$, and then $\Delta f$ and $\Delta f_{H}$ can be considered as constants which are obtained by integrating out the heavy moduli.

The Kähler potential, superpotential, and the uplifting operator are given by

$$
\begin{aligned}
K= & K_{0}\left(t_{V}\right)+Z_{X}\left(t_{V}\right) X^{*} e^{-2 V_{A}} X+Z_{H}\left(t_{V}\right) Q_{H}^{*} e^{2 q V_{A}} Q_{H} \\
& +Z_{H}^{c}\left(t_{V}\right) Q_{H}^{c *} e^{2 q_{c} V_{A}} Q_{H}^{c}+Z_{i}\left(t_{V}\right) Q^{i *} e^{2 q_{i} V_{A}} Q^{i} \\
W= & \omega_{0}+\lambda X^{q+q_{c}} Q_{H}^{c} Q_{H}+\left(N_{c}-N_{f}\right)\left(\frac{e^{-8 \pi^{2} f_{H}}}{\operatorname{det}\left(Q_{H}^{c} Q_{H}\right)}\right)^{\frac{1}{N_{c}-N_{f}}} \\
& +\frac{1}{6} \lambda_{i j k} X^{q_{i}+q_{j}+q_{k}} Q^{i} Q^{j} Q^{k} \\
\mathcal{P}= & \mathcal{P}\left(t_{V}\right),
\end{aligned}
$$

where $t_{V}=T+T^{*}-\delta_{G S} V_{A}, w_{0}$ is a constant of $\mathcal{O}\left(m_{3 / 2} M_{P l}^{2}\right), \lambda$ and $\lambda_{i j k}$ are constant Yukawa couplings, $N_{f}$ denotes the number of flavors for the hidden matter $Q_{H}+Q_{H}^{c}$, $\mathcal{P} \Lambda^{2} \bar{\Lambda}^{2}$ is the uplifting Goldstino superfield operator induced by anti-brane, and finally the $U(1)_{A}$ charge of $X$ is normalized as $q_{X}=-1$. As we have discussed in section 2, anti-brane in KKLT compactification is expected to be sequestered from the $D$-brane of $U(1)_{A}$, and then $\mathcal{P}$ is independent of $t_{V}$. Here we consider more general case that $\mathcal{P}$ can depend on $t_{V}$ in order to see what would be the consequence of the uplifting operator if it is not sequestered from $U(1)_{A}$. Note that the GS cancellation of the mixed anomalies of $U(1)_{A}$ requires

$$
\frac{\delta_{G S}}{2}=\frac{N_{f}\left(q+q^{c}\right)}{8 \pi^{2} k_{H}}=\frac{\sum_{i} q_{i} \operatorname{Tr}\left(T_{a}^{2}\left(Q^{i}\right)\right)}{4 \pi^{2} k_{a}} .
$$

In our case, the non-perturbative superpotential in (4.2), i.e. the Affleck-Dine-Seiberg superpotential [36]

$$
W_{\mathrm{ADS}}=\left(N_{c}-N_{f}\right)\left(\frac{e^{-8 \pi^{2} f_{H}}}{\operatorname{det}\left(Q_{H}^{c} Q_{H}\right)}\right)^{\frac{1}{N_{c}-N_{f}}}
$$

requires a more careful interpretation. If $\lambda$ is so small that the tree level mass $M_{Q}=$ $\lambda\left\langle X^{q+q_{c}}\right\rangle$ of $Q_{H}+Q_{H}^{c}$ is lower than the dynamical scale of $S U\left(N_{c}\right)$ gauge interaction, $W_{\text {ADS }}$ can be interpreted as the non-perturbative superpotential of the light composite meson superfields $\Sigma=Q_{H}^{c} Q_{H}$. However a more plausible possibility is that $\lambda=\mathcal{O}(1)$, and so (in the unit with $M_{P l}=1$ )

$$
M_{Q}=\lambda\left\langle X^{q+q_{c}}\right\rangle \gg \Lambda_{H}=\left(e^{-8 \pi^{2} f_{H}} \operatorname{det}\left(M_{Q}\right)\right)^{1 /\left(3 N_{c}\right)} .
$$

Note that $|X|^{2}=\mathcal{O}\left(\left|\xi_{F I}\right|\right)=\mathcal{O}\left(M_{P l}^{2} / 8 \pi^{2}\right)$ in this model. In this case, the correct procedure to deal with $S U\left(N_{c}\right)$ dynamics is to integrate out first the heavy $Q_{H}+Q_{H}^{c}$ at the scale $M_{Q}$. The resulting effective theory is a pure super YM theory at the scale just below $M_{Q}$, but with the modified gauge kinetic function:

$$
f_{\text {eff }}\left(M_{Q}\right)=f_{H}+\frac{3 N_{c}-N_{f}}{8 \pi^{2}} \ln \left(M_{Q} / M_{P l}\right) .
$$


Then the $S U\left(N_{c}\right)$ gaugino condensation is formed at $\Lambda_{H}$ by this pure super YM dynamics, yielding a non-perturbative superpotential

$$
W_{\text {eff }}=N_{c} M_{Q}^{3} e^{-8 \pi^{2} f_{\text {eff }}\left(M_{\Phi}\right) / N_{c}}
$$

This $W_{\text {eff }}$ is the same as the non-perturbative superpotential obtained by integrating out $\Sigma=Q_{H}^{c} Q_{H}$ using the equations of motion $\partial_{\Sigma} W=0$ for the superpotential of (4.2). In the following, we will simply use the superpotential of (4.2) since it leads to the correct vacuum configuration independently of the value of $M_{Q} / \Lambda_{H}$.

To examine the vacuum configuration of the model (4.2), it is convenient to estimate first the mass scales of the model. As long as $m_{3 / 2}$ is hierarchically smaller than $M_{P l}$, one easily finds that the following mass patterns are independent of the details of SUSY breaking. First, $T$ is stabilized at a vacuum expectation value of $\mathcal{O}(1)$, and as a result

$$
R=\frac{\xi_{F I}}{M_{G S}^{2}}=\frac{2 \partial_{T} K_{0}}{\delta_{G S} \partial_{T} \partial_{\bar{T}} K_{0}}=\mathcal{O}\left(8 \pi^{2}\right)
$$

The $U(1)_{A}$ gauge boson mass-square is dominated by the contribution from $|X|^{2} \sim\left|\xi_{F I}\right|$ :

$$
\frac{M_{A}^{2}}{2 g_{A}^{2}} \simeq Z_{X}|X|^{2}+M_{G S}^{2} \simeq Z_{X}|X|^{2} .
$$

The hidden $S U\left(N_{C}\right)$ confines at the scale

$$
\Lambda_{H}=\left(e^{-8 \pi^{2}\left(k_{H} T+\Delta f_{H}\right)} \operatorname{det}\left(M_{Q} / M_{P l}\right)\right)^{1 /\left(3 N_{c}\right)} M_{P l},
$$

and the $S U\left(N_{c}\right) D$-flat directions of the hidden matter fields are stabilized at

$$
\left\langle Q_{H}^{c} Q_{H}\right\rangle \sim \frac{\Lambda_{H}^{3}}{M_{Q}} .
$$

Finally the hidden $S U\left(N_{c}\right)$ scale and $m_{3 / 2}$ obey the standard relation:

$$
\frac{\Lambda_{H}^{3}}{M_{P l}^{3}} \sim \frac{m_{3 / 2}}{M_{P l}}
$$

It is straightforward to see that in the absence of the uplifting Goldstino operator $\mathcal{P} \Lambda^{2} \bar{\Lambda}^{2}$, the model (4.2) has a unique and stable SUSY AdS vacuum. ${ }^{8}$ For $m_{3 / 2}$ hierarchically smaller than $M_{A}$ and $\Lambda_{H}$, adding the uplifting operator with $\mathcal{P} \sim m_{3 / 2}^{2} M_{P l}^{2}$ triggers a small shift of vacuum configuration, leading to non-zero vacuum expectation values of $F^{T}, F^{X}, F^{\Sigma}$ and $D_{A}$, where $\Sigma=Q_{H}^{c} Q_{H}$. In the following, we compute these SUSY breaking vacuum values within a perturbative expansion in

$$
\frac{\delta_{G S}}{T+T^{*}}=\mathcal{O}\left(\frac{1}{8 \pi^{2}}\right)
$$

\footnotetext{
${ }^{8}$ This SUSY AdS vacuum is a saddle point solution of $V_{F}$, but is the global minimum of $V_{F}+V_{D}$.
} 
while ignoring the corrections suppressed by the following scale hierarchy factors:

$$
\frac{\Lambda_{H}}{M_{A}}, \frac{m_{3 / 2}}{\Lambda_{H}}, \frac{m_{3 / 2}}{M_{\Phi}}, \frac{\left\langle Q_{H}^{c} Q_{H}\right\rangle}{\left\langle X X^{*}\right\rangle} \ll \frac{1}{8 \pi^{2}} .
$$

Let us now examine the vacuum configuration in more detail. As we have mentioned, the true vacuum configuration is given by a small shift induced by $V_{\text {lift }}$ from the SUSY AdS solution of $D_{A}=0$ and $D_{I} W=0$. With this observation, we find (in the unit with $\left.M_{P l}=1\right)$ :

$$
\begin{aligned}
|X|^{2} & =-\frac{\delta_{G S} \partial_{T} K_{0}}{2 Z_{X}}\left(1+\mathcal{O}\left(\frac{1}{8 \pi^{2}}\right)\right) \\
Q_{H}^{c} Q_{H} & =e^{-8 \pi^{2}\left(k_{H} T+\Delta f_{H}\right) / N_{c}}\left(\lambda X^{q+q_{c}}\right)^{\left(N_{f}-N_{c}\right) / N_{c}} \\
\operatorname{Re}(T) & =\frac{N_{c}}{8 \pi^{2} k_{H}} \ln \left|\frac{8 \pi^{2} k_{H}}{\omega_{0} \partial_{T} K_{0}}\right|-\frac{\Delta f_{H}+\Delta f_{H}^{*}}{2 k_{H}}+\frac{N_{f}}{8 \pi^{2} k_{H}} \ln \left|\lambda X^{q+q_{c}}\right|+\mathcal{O}\left(\frac{1}{8 \pi^{2}}\right) \\
& =\frac{N_{c}}{8 \pi^{2} k_{H}} \ln \left(\frac{M_{P l}}{m_{3 / 2}}\right)-\frac{\Delta f_{H}+\Delta f_{H}^{*}}{2 k_{H}}+\mathcal{O}\left(\frac{1}{8 \pi^{2}}\right) .
\end{aligned}
$$

Note that $|X|^{2}=\mathcal{O}\left(M_{P l}^{2} / 8 \pi^{2}\right)$, thus an effect further suppressed by $|X|^{2} / M_{P l}^{2}$ is comparable to the loop correction. The above result on the vacuum expectation value of $\operatorname{Re}(T)$ shows that the GS modulus is stabilized at a value of $\mathcal{O}(1)$ for the model parameters giving the weak scale SUSY, e.g. $m_{3 / 2} \lesssim \mathcal{O}\left(8 \pi^{2}\right) \mathrm{TeV}$.

If $\operatorname{Re}(T)$ is stabilized at a value of $\mathcal{O}(1)$ as desired, $\Sigma=Q_{H}^{c} Q_{H}$ is hierarchically smaller than $M_{P l}^{2}$. Since $F^{\Sigma}=\mathcal{O}\left(\Sigma F^{T}\right)$ and the couplings between $Q_{H}+Q_{H}^{c}$ and the visible fields are suppressed by $1 / M_{P l}$, the contribution from $F^{\Sigma}$ to the visible soft terms can be ignored. Then the soft terms of visible fields are determined by the following four SUSY-breaking auxiliary components:

$$
\begin{aligned}
\frac{F^{T}}{T+T^{*}}= & \frac{m_{3 / 2}^{*}}{8 \pi^{2}}\left(\frac{3 N_{c} \partial_{T} \ln \left(V_{\mathrm{lift}}\right)}{k_{H}\left(T+T^{*}\right) \partial_{T} K_{0}}\right)\left(1+\mathcal{O}\left(\frac{1}{8 \pi^{2}}\right)\right), \\
\frac{F^{X}}{X}= & -F^{T} \partial_{T} \ln \left(-\frac{Z_{X}}{\partial_{T} K_{0}}\right)\left(1+\mathcal{O}\left(\frac{1}{8 \pi^{2}}\right)\right), \\
g_{A}^{2} D_{A}= & \left|F^{T}\right|^{2} \partial_{T} \partial_{\bar{T}} \ln \left(-\frac{Z_{X}}{\partial_{T} K_{0}}\right)\left(1+\mathcal{O}\left(\frac{1}{8 \pi^{2}}\right)\right) \\
& +V_{\text {lift }} \frac{\partial_{T} \ln \mathcal{P}}{\partial_{T} K_{0}}\left(1+\mathcal{O}\left(\frac{1}{8 \pi^{2}}\right)\right), \\
\frac{1}{8 \pi^{2}} \frac{F^{C}}{C_{0}}= & \frac{m_{3 / 2}^{*}}{8 \pi^{2}}\left(1+\mathcal{O}\left(\frac{1}{8 \pi^{2}}\right)\right)
\end{aligned}
$$

where $F^{C} / 8 \pi^{2}$ and $F^{T}$ are the order parameters of anomaly mediation and GS modulus mediation, respectively, and $F^{X}$ and $D_{A}$ are the order parameters of $U(1)_{A}$ mediation. Note that $V_{A}$ and $X$ constitute a massive vector superfield $\tilde{V}_{A}=V_{A}-\ln |X|$. The results on $F^{X}$ and $D_{A}$ can be obtained from eqs. (3.24) and (3.25), while the result on $F^{T}$ can be obtained by applying eqs. (2.28) and (2.31). 
The above results show that generically the GS modulus mediation, the anomaly mediation and the $X$ mediation are comparable to each other. If anti-brane and the $D$ brane of $U(1)_{A}$ are separated from each other by a warped throat, it is expected that $\partial_{T} \ln \mathcal{P}=0$. Then the $U(1)_{A} D$-term mediation is also generically comparable to the other mediations. However, if the Kähler potential of $T$ and $X$ has a special form to give $Z_{X} / \partial_{T} K_{0}=$ constant, we have $F^{X} / X=\mathcal{O}\left(F^{T} / 8 \pi^{2}\right)$ and $D_{A}=\mathcal{O}\left(\left|F^{T}\right|^{2} / 8 \pi^{2}\right)$, thus the $U(1)_{A}$ mediation is suppressed by a loop factor of $\mathcal{O}\left(1 / 8 \pi^{2}\right)$ compared to the GS-modulus and anomaly mediations. Finally, if anti-brane is not sequestered, the resulting $D_{A}$ is of $\mathcal{O}\left(m_{3 / 2}^{2}\right)=\mathcal{O}\left(\left(8 \pi^{2} F^{T}\right)^{2}\right)$ and then soft sfermion masses are dominated by the $U(1)_{A} D$ term contribution. Another important feature of (4.15) is that $F^{T}, F^{X} / X$ and $F^{C} / C_{0}$ are relatively real since $K_{0}, Z_{X}, \mathcal{P}$ are real functions of the real variable $t=T+T^{*}$. As a result, the gaugino masses and $A$-parameters mediated by these auxiliary components automatically preserve CP [37]. Since one can always make $m_{3 / 2}=e^{K / 2} W$ to be real by an appropriate $R$-transformation, all of the above auxiliary components can be chosen to be real, which will be taken in the following discussions.

Applying the above results to the soft terms of (2.35) and also taking into account that $|X|^{2} / M_{P l}^{2}=\mathcal{O}\left(1 / 8 \pi^{2}\right)$, we find the soft masses at the scale just below $M_{G U T}$ :

$$
\begin{aligned}
M_{a}= & M_{0}+\frac{b_{a}}{8 \pi^{2}} g_{G U T}^{2} m_{3 / 2}+\mathcal{O}\left(\frac{M_{0}}{8 \pi^{2}}\right), \\
A_{i j k}= & M_{0}\left(a_{i}+a_{j}+a_{k}\right)-\frac{1}{16 \pi^{2}}\left(\gamma_{i}+\gamma_{j}+\gamma_{k}\right) m_{3 / 2}+\mathcal{O}\left(\frac{M_{0}}{8 \pi^{2}}\right), \\
m_{i}^{2}= & c_{i} M_{0}^{2}-\frac{1}{32 \pi^{2}} \dot{\gamma}_{i} m_{3 / 2}^{2}+\frac{m_{3 / 2} M_{0}}{8 \pi^{2}}\left(\frac{1}{2} \sum_{j k}\left|y_{i j k}\right|^{2}\left(a_{i}+a_{j}+a_{k}\right)-2 C_{2}\left(Q^{i}\right)\right) \\
& -3 q_{i} m_{3 / 2}^{2} \frac{\partial_{T} \ln \mathcal{P}}{\partial_{T} K_{0}}\left(1+\mathcal{O}\left(\frac{1}{8 \pi^{2}}\right)\right)+\mathcal{O}\left(\frac{M_{0}^{2}}{8 \pi^{2}}\right),
\end{aligned}
$$

where $M_{0}$ is the universal modulus-mediated gaugino mass at $M_{G U T}$ :

$$
M_{0} \equiv F^{T} \partial_{T} \ln \operatorname{Re}\left(f_{a}\right)=\frac{m_{3 / 2}}{8 \pi^{2}}\left(\frac{3 N_{c} \partial_{T} \ln V_{\text {lift }}}{k_{H} \partial_{T} K_{0}}\right) \partial_{T} \ln \left(\operatorname{Re}\left(f_{a}\right)\right),
$$

for

$$
\partial_{T} \ln \left(\operatorname{Re}\left(f_{a}\right)\right)=\frac{1}{T+T^{*}+\left(\Delta f+\Delta f^{*}\right) / k}=\frac{k g_{G U T}^{2}}{2},
$$

and

$$
\begin{aligned}
a_{i} & =\frac{\partial_{T} \ln \left(e^{-K_{0} / 3} Z_{i}\left(-Z_{X} / \partial_{T} K_{0}\right)^{q_{i}}\right)}{\partial_{T} \ln \left(\operatorname{Re}\left(f_{a}\right)\right)}, \\
c_{i} & =-\frac{\partial_{T} \partial_{\bar{T}} \ln \left(e^{-K_{0} / 3} Z_{i}\left(-Z_{X} / \partial_{T} K_{0}\right)^{q_{i}}\right)}{\left[\partial_{T} \ln \left(\operatorname{Re}\left(f_{a}\right)\right)\right]^{2}} .
\end{aligned}
$$

Here $\dot{\gamma}_{i}=d \gamma_{i} / d \ln \mu$ for the anomalous dimension $\gamma_{i}=8 \pi^{2} d \ln Z_{i} / d \ln \mu, 2 C_{2}\left(Q^{i}\right)$ is the gauge contribution to $\gamma_{i}$, i.e. $C_{2}\left(Q^{i}\right) \mathbf{1}=\sum_{a} g_{a}^{2} T_{a}^{2}\left(Q^{i}\right)$ for the gauge generator $T_{a}\left(Q^{i}\right)$, 
and finally the canonical Yukawa couplings are given by

$$
y_{i j k}=\frac{\lambda_{i j k}\left(\delta_{G S} / 2\right)^{\left(q_{i}+q_{j}+q_{k}\right) / 2}}{\sqrt{\left(-Z_{X} / \partial_{T} K_{0}\right)^{q_{i}+q_{j}+q_{k}} e^{-K_{0}} Z_{i} Z_{j} Z_{k}}} .
$$

The soft parameters of (4.16) show that the gaugino masses $M_{a}$ in models of KKLT stabilization of the GS modulus are determined by the GS modulus mediation and the anomaly mediation which are comparable to each other. In case that anti-brane is sequestered from $U(1)_{A}$ and thus from the GS modulus $T$, i.e. $\partial_{T} \ln \mathcal{P}=0$, soft sfermion masses are comparable to the gaugino masses. However if anti-brane is not sequestered, soft sfermion mass-squares (for $q_{i} \neq 0$ ) are dominated by the $U(1)_{A} D$-term contribution of $\mathcal{O}\left(8 \pi^{2} M_{a}^{2}\right)$, which might enable us to realize the more minimal supersymmetric standard model scenario [15].

It has been noticed that the low energy gaugino masses obtained from the renormalization group (RG) running of the gaugino masses of (4.16) at $M_{G U T}$ are given by

$$
M_{a}(\mu)=M_{0}\left[1-\frac{1}{4 \pi^{2}} b_{a} g_{a}^{2}(\mu) \ln \left(\frac{M_{G U T}}{\left(M_{P l} / m_{3 / 2}\right)^{\alpha / 2} \mu}\right)\right],
$$

which are same as the low energy gaugino masses in pure modulus-mediation started from the mirage messenger scale

$$
M_{\text {mirage }}=\left(m_{3 / 2} / M_{P l}\right)^{\alpha / 2} M_{G U T}
$$

where

$$
\alpha \equiv \frac{m_{3 / 2}}{M_{0} \ln \left(M_{P l} / m_{3 / 2}\right)}
$$

Similar mirage mediation pattern arises also for the low energy soft sfermion masses if the involved Yukawa couplings are small or $a_{i}+a_{j}+a_{k}=1$ and $c_{i}+c_{j}+c_{k}=1$ for the combination $(i, j, k)=\left(H_{u}, t_{L}, t_{R}\right)$ in the top-quark Yukawa coupling. From (4.17), we find that the anomaly to modulus mediation ratio $\alpha$ is given by

$$
\alpha=\frac{2 \partial_{T} K_{0}}{2 \partial_{T} K_{0}+3 \partial_{T} \ln \mathcal{P}}\left(1+\frac{4 \pi^{2}\left[k_{H}\left(\Delta f+\Delta f^{*}\right)-k\left(\Delta f_{H}+\Delta f_{H}^{*}\right)\right]}{k N_{c} \ln \left(M_{P l} / m_{3 / 2}\right)}\right) .
$$

In the minimal KKLT model, anti-brane is sequestered, i.e $\partial_{T} \ln \mathcal{P}=0, \Delta f \simeq 0$ and $\Delta f_{H} \simeq 0$, thus $\alpha=1$. However in more generic compactifications, it is possible that the gauge kinetic functions $f_{a}$ and $f_{H}$ are given by different linear combinations of $T$ and other moduli. Stabilizing the other moduli can give rise to sizable $\Delta f_{H}$ and/or $\Delta f$, thus a different value of $\alpha$ even in the case that anti-brane is sequestered [38]. In this regard, one interesting possibility is to have $\alpha=2$ which leads to the $\mathrm{TeV}$ scale mirage mediation. As was noticed in [26], the little hierarchy problem of the MSSM can be significantly ameliorated in the $\mathrm{TeV}$ scale mirage mediation scenario. For the model under discussion, $\alpha=2$ can be achieved for instance when $\partial_{T} \ln \mathcal{P}=0, \operatorname{Re}(\Delta f)=0$ and $\operatorname{Re}\left(\Delta f_{H}\right)=-N_{c} / 2$. 
To be more concrete, let us consider the following Kähler potential and the uplifting operator which are expected to be valid for a wide class of string compactifications:

$$
\begin{aligned}
f_{a} & =k T, \quad f_{H}=k_{H} T+\Delta f_{H}, \\
K_{0} & =-n_{0} \ln \left(t_{V}\right), \quad Z_{I}=\frac{1}{t_{V}^{n_{I}}}, \quad \mathcal{P}=\frac{\mathcal{P}_{0}}{t_{V}^{n_{P}}},
\end{aligned}
$$

where $Z_{I}$ denote the Kähler metric of $\Phi^{I}=\left(X, Q^{i}\right)$, and $\mathcal{P}_{0}$ is a constant of $\mathcal{O}\left(m_{3 / 2}^{2} M_{P l}^{2}\right)$. Applying (4.14), (4.15), (4.16) and (4.24) to this form of gauge kinetic functions, Kähler potential and uplifting operator, we find

$$
\begin{aligned}
|X|^{2} & =\frac{n_{0} \delta_{G S}}{2\left(T+T^{*}\right)^{1-n_{X}}}, \\
\frac{F^{X}}{X} & =\left(n_{X}-1\right) M_{0}, \\
g_{A}^{2} D_{A} & =\left(n_{X}-1\right) M_{0}^{2}+\frac{3 n_{P}}{n_{0}} m_{3 / 2}^{2}, \\
a_{i} & =c_{i}=\frac{1}{3} n_{0}-n_{i}-\left(n_{X}-1\right) q_{i}, \\
\alpha & =\frac{2 n_{0}}{2 n_{0}+3 n_{P}}\left(1-\frac{4 \pi^{2}\left(\Delta f_{H}+\Delta f_{H}^{*}\right)}{N_{c} \ln \left(M_{P l} / m_{3 / 2}\right)}\right), \\
y_{i j k} & =\frac{\left(n_{0} \delta_{G S} / 2\right)^{\left(q_{i}+q_{j}+q_{k}\right) / 2}}{\left(T+T^{*}\right)^{\left(a_{i}+a_{j}+a_{k}\right) / 2} \lambda_{i j k} .}
\end{aligned}
$$

In fact, since $U(1)_{A}$ is spontaneously broken by $\langle X\rangle \sim M_{P l} / \sqrt{8 \pi^{2}}$, the soft parameters of (4.16) can be obtained also from an effective SUGRA which would be derived by integrating out the massive vector multiplet $\tilde{V}_{A}=V_{A}-\ln |X|$ as well as the hidden matter $Q_{H}+Q_{H}^{c}$. To derive the effective SUGRA, it is convenient to make the following field redefinition:

$$
\begin{aligned}
V_{A} & \rightarrow V_{A}+\ln |X| \\
T & \rightarrow T+\frac{\delta_{G S}}{2} \ln (X), \\
Q^{I} & \rightarrow X^{-q_{I}} Q^{I} \quad\left(Q^{I}=Q_{H}, Q_{H}^{c}, Q^{i}\right) .
\end{aligned}
$$

This field redefinition induces an anomalous variation of the gauge kinetic functions

$$
\begin{aligned}
& f_{a} \rightarrow f_{a}-\frac{1}{4 \pi^{2}} \sum_{i} q_{i} \operatorname{Tr}\left(T_{a}^{2}\left(Q^{i}\right)\right) \ln (X), \\
& f_{H} \rightarrow f_{H}-\frac{1}{8 \pi^{2}}\left(q+q^{c}\right) N_{f} \ln (X) .
\end{aligned}
$$

Taking into account this change of gauge kinetic functions together with the anomaly cancellation condition (4.3), the model in the new field basis is given by

$$
\begin{aligned}
K & =K_{0}\left(t_{V}\right)+Z_{X}\left(t_{V}\right) e^{-2 V_{A}}+Z_{I}\left(t_{V}\right) Q^{I *} e^{2 q_{I} V_{A}} Q^{I} \\
W & =\omega_{0}+\lambda Q_{H}^{c} Q_{H}+\frac{1}{6} \lambda_{i j k} Q^{i} Q^{j} Q^{k}, \\
f_{a} & =k T+\Delta f, \quad f_{H}=k_{H} T+\Delta f_{H}, \quad \mathcal{P}=\mathcal{P}\left(t_{V}\right),
\end{aligned}
$$


In the new field basis, $V_{A}$ corresponds to the massive vector superfield $\tilde{V}_{A}$. The heavy hidden matter $Q_{H}+Q_{H}^{c}$ can be easily integrated out, leaving a threshold correction to the hidden gauge kinetic function: $\delta f_{H}=-N_{f} \ln (\lambda) / 8 \pi^{2}$. The massive vector superfield can be also integrated out using the equation of motion:

$$
\frac{\partial K}{\partial V_{A}}-\theta^{2} \bar{\theta}^{2} C C^{*} e^{K / 3} \frac{\partial \mathcal{P}}{\partial V_{A}}=0 .
$$

For simplicity, here we will consider only the case of sequestered anti-brane, i.e. $\partial \mathcal{P} / \partial V_{A}=$ 0 . The generalization to unsequestered anti-brane is straightforward. Making an expansion in $\delta_{G S}=\mathcal{O}\left(1 / 8 \pi^{2}\right)$, the solution of the above equation is given by

$$
\begin{aligned}
e^{-2 V_{A}}= & -\frac{\delta_{G S} \partial_{T} K_{0}}{2 Z_{X}}\left(1+\mathcal{O}\left(\frac{1}{8 \pi^{2}}\right)\right) \\
& +q_{i}\left(\frac{-2 Z_{X}}{\delta_{G S} \partial_{T} K_{0}}\right)^{q_{i}}\left(1+\mathcal{O}\left(\frac{1}{8 \pi^{2}}\right)\right) Z_{i} Q^{i *} Q^{i} .
\end{aligned}
$$

Inserting this solution to 4.29) and also adding the gaugino condensation superpotential of the super $S U\left(N_{c}\right)$ YM theory whose gauge kinetic function is now given by $f_{H}=$ $k_{H} T+\Delta f_{H}+\delta f_{H}$, we find the following effective SUGRA:

$$
\begin{aligned}
K_{\mathrm{eff}} & =K_{0}(t)+\left|\frac{Z_{X}(t)}{\partial_{T} K_{0}(t)}\right|^{q_{i}} Z_{i}(t) Q^{i *} Q^{i}, \\
W_{\mathrm{eff}} & =w_{0}+N_{c} \lambda^{N_{f} / N_{c}} e^{-8 \pi^{2} \Delta f_{H} / N_{c}} e^{-8 \pi^{2} k_{H} T / N_{c}}+\frac{1}{6}\left|\delta_{G S} / 2\right|^{\left(q_{i}+q_{j}+q_{k}\right) / 2} \lambda_{i j k} Q^{i} Q^{j} Q^{k}, \\
f_{a}^{\mathrm{eff}} & =k T+\Delta f, \quad \mathcal{P}_{\mathrm{eff}}=\mathcal{P}=\mathrm{constant},
\end{aligned}
$$

where $t=T+T^{*}$ and we made the final field redefinition $Q^{i} \rightarrow\left|\delta_{G S} / 2\right|^{q_{i} / 2} Q^{i}$. One can now compute the vacuum values of $T, F^{T}$ and the resulting soft terms of visible fields using the above effective SUGRA, and finds the same results as those in (4.14), (4.15) and (4.16) for $\partial_{T} \ln \mathcal{P}=0$.

\section{Conclusion}

In this paper, we examined the effects of anomalous $U(1)_{A}$ gauge symmetry on SUSY breaking while incorporating the stabilization of the modulus-axion multiplet responsible for the GS anomaly cancellation mechanism. Since our major concern is the KKLT stabilization of the GS modulus, we also discussed some features such as the $D$-type spurion dominance and the sequestering of the SUSY breaking by red-shifted anti-brane which is a key element of the KKLT moduli stabilization. It is noted also that the $U(1)_{A} D$-term potential can not be an uplifting potential for dS vacuum in SUSY breaking scenarios with a gravitino mass hierarchically smaller than the Planck scale. In case of the KKLT stabilization of the GS modulus, soft terms of visible fields are determined by the GS modulus mediation, the anomaly mediation and the $U(1)_{A}$ mediation which are generically comparable to each other, thereby yielding the mirage mediation pattern of the superparticle masses at low energy scale. 


\section{Acknowledgments}

We thank A. Casas, T. Kobayashi, K.-I. Okumura for useful discussions, and particularly Ian Woo Kim for explaining us some features of the supersymmetry breaking by red-shifted anti-brane which are presented in this paper. This work is supported by the KRF Grant funded by the Korean Government (KRF-2005-201-C00006), the KOSEF Grant (KOSEF R01-2005-000-10404-0), and the Center for High Energy Physics of Kyungpook National University. K.S.J acknowledges also the support of the Spanish Government under the Becas MAEC-AECI, Curso 2005/06.

\section{Appendix A. SUSY breaking by red-shifted anti-brane}

In this appendix, we discuss the red-shift of the couplings of $4 \mathrm{D}$ graviton and gravitino on the world volume of anti-brane within the framework of the supersymmetric RandallSundrum model on $S^{1} / Z_{2}$ [39]. The bulk action of the model is given by

$$
\begin{aligned}
S_{5 \mathrm{D}}= & -\frac{1}{2} \int d^{4} x d y \sqrt{-G} M_{5}^{3}\left\{R_{5}+\bar{\Psi}_{M}^{i} \gamma^{M N P} D_{N} \Psi_{i P}\right. \\
& \left.-\frac{3}{2} k \epsilon(y) \bar{\Psi}_{M}^{i} \gamma^{M N}\left(\sigma_{3}\right)_{i j} \Psi_{N}^{j}-12 k^{2}+\frac{(\delta(y)-\delta(y-\pi))}{\sqrt{G_{55}}} 12 k\right\},
\end{aligned}
$$

where $R_{5}$ is the 5D Ricci scalar for the metric $G_{M N}, \Psi_{M}^{i}(i=1,2)$ are the symplectic Majorana gravitinos, $M_{5}$ is the 5 -dimensional Planck scale, and $k$ is the AdS curvature. Here we have ignored the graviphoton as it is not relevant for our discussion. The relations between the gravitino kink mass and the brane cosmological constants are determined by supersymmetry. Imposing the standard orbifold boundary conditions on the 5-bein and $5 \mathrm{D}$ gravitino, one finds that a slice of $\mathrm{AdS}_{5}$ is a solution of the equations of motion:

$$
d s^{2}=e^{-2 k L|y|} \eta_{\mu \nu} d x^{\mu} d x^{\nu}+L^{2} d y^{2} \quad(-\pi \leq y \leq \pi)
$$

where $L$ is the orbifold radius. The corresponding gravitino zero mode equation is given by

$$
\partial_{y} \Psi_{(0) \mu}^{i}+\frac{L}{2} k \epsilon(y)\left(\sigma_{3}\right)^{i}{ }_{j} \gamma_{5} \Psi_{(0) \mu}^{j}=0
$$

yielding the following 4D graviton and gravitino zero modes:

$$
\begin{aligned}
G_{(0) \mu \nu}(x, y) & =e^{-2 k L|y|} g_{\mu \nu}(x), \\
\Psi_{(0)_{\mu}}^{i=1}(x, y) & =e^{-\frac{1}{2} k L|y|} \psi_{\mu L}(x) .
\end{aligned}
$$

The above form of wavefunctions reflects the quasi-localization of the $4 \mathrm{D}$ graviton and gravitino zero modes at the UV fixed point $y=0$, leading to a red-shift of the zero mode

couplings at the IR fixed point $y=\pi$. To make an analogy with the KKLT set-up, let us introduce a brane of $4 \mathrm{D}$ AdS SUGRA at $y=0$ and an anti-brane of non-linearly realized 
4D SUGRA at $y=\pi$. Written in terms of the $4 \mathrm{D}$ zero modes $g_{\mu \nu}(x)$ and $\psi_{\mu}$, the UV brane action is given by

$$
\begin{aligned}
S_{\mathrm{UV}}= & \int d^{4} x \sqrt{-g}\left[3 m_{\mathrm{UV}}^{2} M_{0}^{2}-\frac{1}{2} M_{0}^{2} R(g)\right. \\
& \left.-\frac{1}{2}\left(\epsilon^{\mu \nu \rho \sigma} \bar{\psi}_{\mu} \gamma_{5} \gamma_{\nu} D_{\rho} \psi_{\sigma}+m_{\mathrm{UV}} \bar{\psi}_{\mu L} \sigma^{\mu \nu} \psi_{\nu R}+\text { h.c. }\right)\right] .
\end{aligned}
$$

As for the anti-brane action with non-linearly realized 4D SUGRA at $y=\pi$, let us choose the unitary gauge of $\xi^{\alpha}=0$, where $\xi^{\alpha}$ is the Goldstino fermion living on the world-volume of anti-brane. Then using

$$
\begin{aligned}
G_{(0) \mu \nu}(x, \pi) & =e^{-2 \pi k L} g_{\mu \nu}(x), \\
\Psi_{(0)_{\mu}}^{i=1}(x, \pi) & =e^{-\pi k L / 2} \psi_{\mu L}(x),
\end{aligned}
$$

one easily finds that a generic anti-brane action of $g_{\mu \nu}$ and $\psi_{\mu}$ can be written as 40.

$$
\begin{aligned}
S_{\mathrm{IR}}= & \int d^{4} x \sqrt{-g}\left[-e^{4 A} \Lambda_{1}^{4}-\frac{1}{2} e^{2 A} \Lambda_{2}^{2} R(g)+e^{2 A} Z_{1} \epsilon^{\mu \nu \rho \sigma} \bar{\psi}_{\mu} \gamma_{5} \gamma_{\nu} D_{\rho} \psi_{\sigma}\right. \\
& +e^{3 A}\left(\Lambda_{3} \bar{\psi}_{\mu L} \sigma^{\mu \nu} \psi_{\nu R}+\Lambda_{4} \bar{\psi}_{\mu L} \psi_{R}^{\mu}+\text { h.c. }\right) \\
& \left.+e^{2 A} \bar{\psi}_{\mu} \gamma_{5} \gamma_{\nu} D_{\rho} \psi_{\sigma}\left(Z_{2} g^{\mu \nu} g^{\rho \sigma}+Z_{3} g^{\mu \rho} g^{\nu \sigma}+Z_{4} g^{\mu \sigma} g^{\nu \rho}\right)\right],
\end{aligned}
$$

where $e^{A} \equiv e^{-\pi k L}$ and all the coefficients, i.e. $\Lambda_{i}$ and $Z_{i}(i=1, . ., 4)$, are of order unity in the unit with $M_{5}=1$.

In fact, adding the brane actions (5.4) and (5.6) to the bulk action (5.1) makes the solution (5.3) unstable. This problem can be avoided by introducing a proper mechanism to stabilze the orbifold radius $L$. In the KKLT compactifications of Type IIB string theory, such stabilization is achieved by the effects of fluxes. Generalization of (5.1), (5.4) and (5.6) incorporating the stabilization of the radion $L$ will modify the wavefunctions of the graviton and gravitino zero modes, however still (5.3) provides a qualitatively good approximation for the modified wavefunctions as long as the quasi-localization of zero modes is maintained. To compensate the negative vacuum energy density of the UV brane, the anti-brane should provide a positive vacuum energy density: $e^{4 A} \Lambda_{1}^{4} \simeq 3 m_{\mathrm{UV}}^{2} M_{0}^{2}$, which requires $e^{2 A} \sim$ $m_{\mathrm{UV}} / M_{0}$ for $M_{0} \sim \Lambda_{1}$. For this value of the warp factor, the $4 \mathrm{D}$ Planck scale and gravitino mass are given by

$$
M_{P l}^{2} \simeq \frac{M_{5}^{3}}{k}+M_{0}^{2}, \quad m_{3 / 2} \simeq m_{\mathrm{UV}}
$$

where we have assumed $M_{5} \sim k \sim M_{0}$ and ignored the contributions suppressed by an additional power of $e^{A}$. Then one finds that SUSY breaking effects due to the terms of $S_{\mathrm{IR}}$ other than $e^{4 A} \Lambda_{1}^{4}$ are suppressed by more powers of $e^{A} \sim \sqrt{m_{3 / 2} / M_{P l}}$ compared to the effects due to the terms in $S_{5 D}$ and $S_{\mathrm{UV}}$ even when $\Lambda_{i}$ and $Z_{i}$ are all of order unity in the unit with $M_{P l}=1$. For instance, the gravitino mass from $S_{\mathrm{IR}}$ is of $\mathcal{O}\left(e^{3 A} M_{P l}\right)$, while the gravitino mass from $S_{\mathrm{UV}}$ is $m_{3 / 2}=\mathcal{O}\left(e^{2 A} M_{P l}\right)$. 


\section{References}

[1] M. B. Green and J. H. Schwarz, Phys. Lett. B149, 117 (1984).

[2] C. P. Burgess, R. Kallosh and F. Quevedo, JHEP 0310, 056 (2003) [arXiv: hep-th/0309187]

[3] H. Jockers and J. Louis, Nucl. Phys. B718, 203 (2005) [arXiv: hep-th/0502059]

[4] G. Aldazabal, A. Font, L. E. Ibanez and G. Violero, Nucl. Phys. B 536, 29 (1998) [arXiv:hep-th/9804026]; Z. Lalak, S. Lavignac and H. P. Nilles, Nucl. Phys. B 559, 48 (1999) [arXiv: hep-th/9903160].

[5] M. Dine, N. Seiberg and E. Witten, Nucl. Phys. B289, 589 (1987); M. Dine, I. Ichinose and N. Seiberg, Nucl. Phys. B293, 253 (1987).

[6] C. D. Froggatt and H. B. Nielsen, Nucl. Phys. B147, 277 (1979).

[7] L. E. Ibanez and G. G. Ross, Phys. Lett. B 332, 100 (1994) [arXiv: hep-ph/9403338]; V. Jain and R. Shrock, Phys. Lett. B 352, 83 (1995) [arXiv: hep-ph/9412367]; P. Binetruy and P. Ramond, Phys. Lett. B 350, 49 (1995) [arXiv: hep-ph/9412385]; E. Dudas, S. Pokorski and C. A. Savoy, Phys. Lett. B 356, 45 (1995) [arXiv: hep-ph/9504292]; P. Binetruy, S. Lavignac and P. Ramond, Nucl. Phys. B 477, 353 (1996) [arXiv: hep-ph/9601243]; E. J. Chun and A. Lukas, Phys. Lett. B 387, 99 (1996) [arXiv: hep-ph/9605377]; E. Dudas, C. Grojean, S. Pokorski and C. A. Savoy, Nucl. Phys. B 481, 85 (1996)[arXiv: hep-ph/9606383]; K. Choi, E. J. Chun and H. D. Kim, Phys. Lett. B 394, 89 (1997) [arXiv: hep-ph/9611293]; P. H. Chankowski, K. Kowalska, S. Lavignac and S. Pokorski, Phys. Rev. D 71, 055004 (2005) [arXiv: hep-ph/0501071].

[8] Y. Kawamura and T. Kobayashi, Phys. Lett. B375, 141 (1996) [Erratum-ibid. B388, 867 (1996)] [arXiv: hep-ph/9601365]; P. Binetruy and E. Dudas, Phys. Lett. B 389, 503 (1996) [arXiv: hep-th/9607172]; G. R. Dvali and A. Pomarol, Phys. Rev. Lett. 77, 3728 (1996) [arXiv: hep-ph/9607383]; Y. Kawamura and T. Kobayashi, Phys. Rev. D56, 3844 (1997) [arXiv: hep-ph/9608233]; Y. Kawamura, Phys. Lett. B 446, 228 (1999) [arXiv: hep-ph/9811312]; B. Murakami, K. Tobe and J. D. Wells, Phys. Lett. B526, 157 (2002) [arXiv: hep-ph/0111003]; T. Higaki, Y. Kawamura, T. Kobayashi and H. Nakano, Phys. Rev. D 69, 086004 (2004) [arXiv: hep-ph/0308110]; B. Kors and P. Nath, Nucl. Phys. B 711, 112 (2005) [arXiv: hep-th/0411201]; K. S. Babu, T. Enkhbat and B. Mukhopadhyaya, Nucl. Phys. B 720, 47 (2005) [arXiv: hep-ph/0501079].

[9] A. E. Nelson and D. Wright, Phys. Rev. D 56, 1598 (1997) [arXiv:hep-ph/9702359].

[10] N. Arkani-Hamed, M. Dine and S. P. Martin, Phys. Lett. B 431, 329 (1998) [arXiv: hep-ph/9803432]; T. Barreiro, B. de Carlos, J. A. Casas and J. M. Moreno, Phys. Lett. B 445, 82 (1998) [arXiv: hep-ph/9808244].

[11] M. P. Garcia del Moral, JHEP 0604, 022 (2006) [arXiv: hep-th/0506116].

[12] E. Dudas and S. K. Vempati, Nucl. Phys. B 727, 139 (2005) [arXiv: hep-th/0506172]; G. Villadoro and F. Zwirner, Phys. Rev. Lett. 95, 231602 (2005) [arXiv: hep-th/0508167]; S.L. Parameswaran and A. Westphal, hep-th/0602253

[13] A. Achucarro, B. de Carlos, J.A. Casas and L. Doplicher, hep-th/0601190

[14] N. V. Krasnikov, Phys. Lett. B193, 37 (1987); J.A. Casas, Z. Lalak, C. Munoz and G. G. Ross, Nucl. Phys. B347, 243 (1990); T.R. Taylor, Phys. Lett. B252, 59 (1990); B. de Carlos, J.A. Casas and C. Munoz, Nucl. Phys. B399, 623 (1993) [arXiv: hep-th/9204012]. 
[15] K. Choi, J. E. Kim and H. P. Nilles, Phys. Rev. Lett. 73, 1758 (1994) [arXiv: hep-ph/9404311].

[16] T. Banks and M. Dine, Phys. Rev. D50, 7454 (1994) [arXiv: hep-th/9406132]; J. A. Casas, Phys. Lett. B384, 103 (1996) [arXiv: hep-th/9605180]; T. Barreiro, B. de Carlos, E. J. Copeland, Phys. Rev. D57, 7354 (1998) [arXiv: hep-ph/9712443].

[17] S. Kachru, R. Kallosh, A. Linde and S. P. Trivedi, Phys. Rev. D68, 046005 (2003) [arXiv: hep-th/0301240].

[18] S. B. Giddings, S. Kachru and J. Polchinski, Phys. Rev. D66, 106006 (2002) [arXiv: hep-th/0105097].

[19] S. Kachru, J. Pearson and H. Verlinde, JHEP06 (2002) 021 [arXiv: hep-th/0112197].

[20] K. Choi, A. Falkowski, H. P. Nilles, M. Olechowski and S. Pokorski, JHEP 0411, 076 (2004) [arXiv: hep-th/0411066]; K. Choi, A. Falkowski, H. P. Nilles and M. Olechowski, Nucl. Phys. B718, 113 (2005) [arXiv: hep-th/0503216].

[21] O. Lebedev, H. P. Nilles, M. Ratz, Phys. Lett. B636, 126 (2006) [arXiv: hep-th/0603047].

[22] V. S. Kaplunovsky and J. Louis, Phys. Lett. B 306, 269 (1993) [arXiv: hep-th/9303040]; A. Brignole, L. E. Ibanez and C. Munoz, Nucl. Phys. B 422, 125 (1994) [Erratum-ibid. B 436, 747 (1995)] [arXiv: hep-ph/9308271].

[23] L. Randall and R. Sundrum, Nucl. Phys. B 557, 79 (1999) [arXiv: hep-th/9810155]; G. F. Giudice, M. A. Luty, H. Murayama and R. Rattazzi, JHEP 9812, 027 (1998) [arXiv: hep-ph/9810442]; J. A. Bagger, T. Moroi and E. Poppitz, JHEP 0004, 009 (2000) [arXiv: hep-th/9911029]; P. Binetruy, M. K. Gaillard and B. D. Nelson, Nucl. Phys. B 604, 32 (2001) [arXiv: hep-ph/0011081].

[24] K. Choi, K. S. Jeong and K. Okumura, JHEP 0509, 039 (2005) [arXiv: hep-ph/0504037].

[25] M. Endo, M. Yamaguchi and K. Yoshioka, Phys. Rev. D72, 015004 (2005) [arXiv: hep-ph/0504036]; A. Falkowski, O. Lebedev and Y. Mambrini, JHEP 0511, 034 (2005) [arXiv: hep-ph/0507110]; H. Baer, E.-K. Park, X. Tata and T. T. Wang, hep-ph/0604253.

[26] K. Choi, K. S. Jeong, T. Kobayashi and K.-I. Okumura, Phys. Lett. B633, 355 (2006) [arXiv: hep-ph/0508029]; R. Kitano and Y. Nomura, Phys. Lett. B631, 58 (2005) [arXiv: hep-ph/0509039]; O. Lebedev, H. P. Nilles and M. Ratz, hep-ph/0511320; R. Kitano and Y. Nomura, hep-ph/0602096; A. Pierce and J. Thaler, hep-ph/0604192.

[27] E. Poppitz, Nucl. Phys. B542, 31 (1999) [arXiv: hep-th/9810010].

[28] S. Samuel and J. Wess, Nucl. Phys. B221, 153 (1983).

[29] L. Randall and R. Sundrum, Phys. Rev. Lett. 83, 3370 (1999) [arXiv: hep-ph/9905221].

[30] M. A. Luty and R. Sundrum, Phys. Rev. D64, 065012 (2001) [arXiv: hep-th/0012158].

[31] M. Luty and R. Sundrum, Phys. Rev. D67, 045007 (2003) [arXiv: hep-th/0111231]; Phys. Rev. D65, 066004 (2002) [arXiv: hep-th/0105137]; T. Kobayashi, H. Nakano, T. Noguchi and H. Terao, Phys. Rev. D66, 095011 (2002) [arXiv: hep-ph/0202023]; M. Ibe, K.-I. Izawa, Y. Nakayama, Y. Shinbara and T. Yanagida, Phys. Rev. D73, 015004 (2006) [arXiv: hep-ph/0506023].

[32] T. Gregoire, R. Rattazzi, C.A. Scrucca, A. Strumia and E. Trincherini, Nucl. Phys. B720, 3 (2005) [arXiv: hep-th/0411216]; A. Falkowski, JHEP 0505, 073 (2005) [arXiv: hep-th/0502072]. 
[33] I. W. Kim and S. D. Shin, work in preparation.

[34] F. Brummer, A. Hebecker and E. Trincherini, Nucl. Phys. B738, 283 (2006) [arXiv: hep-th/0510113].

[35] Y. Kawamura, Phys. Lett. B 446, 228 (1999) [arXiv: hep-ph/9811312]

[36] I. Affleck, M. Dine and N. Seiberg, Phys. Rev. Lett. 51, 1026 (1983)

[37] K. Choi, Phys. Rev. Lett. 72, 1592 (1994) [arXiv: hep-ph/9311352].

[38] H. Abe, T. Higaki and T. Kobayashi, Phys. Rev. D73, 046005 (2006) [arXiv: hep-th/0511160].

[39] R. Altendorfer, J. Bagger and D. Nemeschansky, Phys. Rev. D63, 125025 (2001) [arXiv: hep-th/0003117]; T. Gherghetta and A. Pomarol, Nucl. Phys. B586, 141 (2000) [arXiv: hep-ph/0003129]; A. Falkowski, Z. Lalak and S. Pokorski, Phys. Lett. B491, 172 (2000) [arXiv: hep-th/0004093]; K. Choi, H. D. Kim and I. W. Kim, JHEP 0211, 033 (2002) [arXiv: hep-ph/0202257].

[40] T.E. Clark, S.T. Love, M. Nitta and T. ter Veldhuis, hep-th/0512078 